JOURNAL OF

SYMPLECTIC GEOMETRY

Volume 12, Number 1, 171-213, 2014

\title{
THE DUISTERMAAT-HECKMAN FORMULA AND THE COHOMOLOGY OF MODULI SPACES OF POLYGONS
}

\author{
Alessia Mandini
}

\begin{abstract}
We give a presentation of the cohomology ring of spatial polygon spaces $M(r)$ with fixed side lengths $r \in \mathbb{R}_{+}^{n}$. These spaces can be described as the symplectic reduction of the Grassmaniann of 2-planes in $\mathbb{C}^{n}$ by the $U(1)^{n}$-action by multiplication, where $U(1)^{n}$ is the torus of diagonal matrices in the unitary group $U(n)$. We prove that the first Chern classes of the $n$ line bundles associated with the fibration $(r$-level set $) \rightarrow M(r)$ generate the cohomology ring $H^{*}(M(r), \mathbb{C})$. By applying the Duistermaat-Heckman Theorem, we then deduce the relations on these generators from the piece-wise polynomial function that describes the volume of $M(r)$. We also give an explicit description of the birational map between $M(r)$ and $M\left(r^{\prime}\right)$ when the lengths vectors $r$ and $r^{\prime}$ are in different chambers of the moment polytope. This wallcrossing analysis is the key step to prove that the Chern classes above are generators of $H^{*}(M(r))$ (this is well-known when $M(r)$ is toric, and by wall-crossing we prove that it holds also when $M(r)$ is not toric).
\end{abstract}

\section{Introduction}

Spatial polygon spaces are a widely studied family of moduli spaces obtained by symplectic reduction, see for example $[$ AG, Go, HK98, HK97, $\mathbf{K T}, \mathbf{K M}, \mathbf{K h}, \mathbf{K l}, \mathbf{K o}, \mathbf{M}, \mathbf{T a 0 1}, \mathbf{T a 0 2}]$. A first way to introduce $M(r)$ is as the space of closed piece-wise linear paths in $\mathbb{R}^{3}$ such that the $j$ th step has norm $r_{j}$, modulo rotations and translations. The vector $r=\left(r_{1}, \ldots, r_{n}\right) \in$ $\mathbb{R}_{+}^{n}$ is called the lengths vector.

Kapovich and Millson $[\mathbf{K M}]$ showed that one can describe $M(r)$ by means of a symplectic quotient as follows. Let $\mathcal{S}_{r}=\prod_{j=1}^{n} S_{r_{j}}^{2}$ be the product of $n$ spheres in $\mathbb{R}^{3}$ of radii $r_{1}, \ldots, r_{n}$ and centers all the origin. The diagonal $S O(3)$-action on $\mathcal{S}_{r}$ is Hamiltonian with moment map $\mu: \mathcal{S}_{r} \rightarrow \mathfrak{s o}(3)^{*} \simeq \mathbb{R}^{3}$, $\mu\left(e_{1}, \ldots, e_{n}\right)=e_{1}+\cdots+e_{n}$. Note that an element $\left(e_{1}, \ldots, e_{n}\right) \in \mathcal{S}_{r}$ is in $\mu^{-1}(0)$ if and only if the path in $\mathbb{R}^{3}$ with edges $e_{1}, \ldots, e_{n}$ closes to a polygon. 
The moduli space of spatial polygons $M(r)$ arises then as the symplectic quotient $\mu^{-1}(0) / S O(3)=: \mathcal{S}_{r} / / 0 S O(3)$.

This fits into a broader picture: let $U(1)^{n}$ be the maximal torus of diagonal matrices in the unitary group $U(n)$ and consider the action by conjugation of $U(1)^{n} \times U(2) \subset U(n) \times U(2)$ on $\mathbb{C}^{n \times 2}$ (an element in the complex space is naturally thought as an $n \times 2$ matrix). Note that the diagonal circle $U(1) \subset U(1)^{n} \times U(2)$ fixes everything, and therefore only the action of $K:=U(1)^{n} \times U(2) / U(1)$ is effective. One can then realize the polygon space $M(r)$ as the symplectic reduction

$$
\mathbb{C}^{n \times 2} / /_{(r, 0)} K
$$

cf. [HK97]. It is enlightening to perform the symplectic reduction in stages. Taking first the quotient by $U(1)^{n}$ one obtains the product of spheres $\mathcal{S}_{r}$ (here the reduction is performed by means of the Hopf map as explained in Section 2 and in [HK97]). The residual $U(2) / U_{1} \simeq S O(3)$ action is the one described above, and one recovers the description of the polygon space $M(r)$ as the symplectic quotient $\mathcal{S}_{r} / /_{0} S O(3)$.

Performing the reduction in stages in the opposite order, one obtains the Gelfand-MacPherson correspondence. In fact, one first obtains the Grassmanian $\operatorname{Gr}(2, n)$ of complex planes in $\mathbb{C}^{n}$ as the reduction $\mathbb{C}^{n \times 2} / /_{0} U(2)$. Then the quotient by the residual $U(1)^{n} / U(1)$ action on $G r(2, n)$ is isomorphic to the moduli space of $n$ points in $\mathbb{C P}^{1}$, cf. $[\mathbf{G M}]$, and hence, by Klyachko $[\mathbf{K l}]$ and Kapovich and Millson [KM], is also isomorphic to the polygon space $M(r)$. This is summarized in the following diagram:

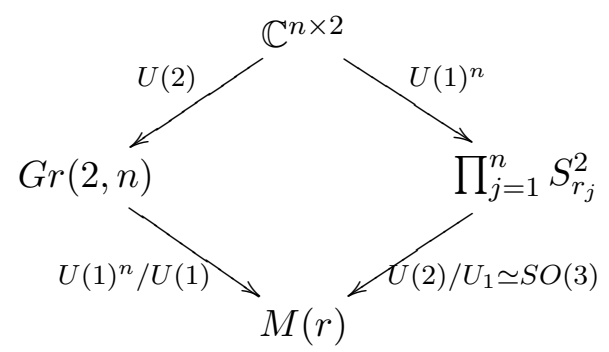

These two descriptions of the moduli space of polygons intertwine throughout the paper, and give rise to the description we present of the cohomology ring of $M(r)$. On the subject there is a broad literature. Hausmann and Knutson [HK98] computed the integer cohomology rings of the moduli spaces $M(r)$ by embedding these spaces (which in general are not toric) in toric varieties and computing the kernel of the induced restriction map on cohomologies. The cohomology ring of the polygon space was also computed by Goldin. In fact, in [Go] she finds explicit formulae for the rational cohomology ring of the symplectic reduction of coadjoint orbits of $S U(n)$ by the action of a maximal torus. Considering degenerate coadjoint orbits 
she determines the cohomology ring of the reduction of the Grassmannian of $k$-planes in $\mathbb{C}^{n}$. By the Gelfand-MacPherson correspondence, see [GM], this is the moduli space of $n$ points in $\mathbb{C P}^{k-1}$ which, for $k=2$, is isomorphic to the moduli space of $n$-sided polygons in $\mathbb{R}^{3}$. Previously Brion $[\mathbf{B}]$ and Kirwan $[\mathbf{K i}]$ have computed the rational cohomology ring of the special case of equilateral polygon spaces $M(1, \ldots, 1)$ with an odd number of edges.

Many other contributed to the study of these spaces; see for example [AG, Ko, Ta01] where the intersection numbers are explicitly computed (by means of a recursion formula in $[\mathbf{A G}]$, using "quantization commutes with reduction" in [Ta01], via an algebro-geometric approach in $[\mathbf{K o}])$. More contextualized reference will be given throughout the paper.

Our approach to the cohomology ring of $M(r)$ is as follows. First, we give an explicit description of the birational map between two polygon spaces $M(r)$ and $M\left(r^{\prime}\right)$ when $r$ and $r^{\prime}$ lie in different chambers of the moment polytope $\mu_{U(1)^{n}}^{-1}(G r(2, n))$. Using this description, we prove that the first Chern classes $c_{1}, \ldots, c_{n}$ of the $n$ line bundles associated to the fibration $\mu_{U(1)^{n}}^{-1}(r) \rightarrow M(r)$ generate the cohomology ring $H^{*}(M(r), \mathbb{C})$ (whenever $M(r)$ is a smooth manifold). This provides the opportunity to determine the relations on the generators $c_{1}, \ldots, c_{n}$ by applying the Duistermaat-Heckman Theorem, once the piece-wise polynomial function $\operatorname{vol} M(r)$ that associates to each generic $r$ the symplectic volume of $M(r)$ is known, as it is in our case. In particular, this also proves that polygon spaces only have even dimensional cohomology. The fact that $H^{*}(M(r), \mathbb{C})$ is generated by the classes $c_{i}$ 's was established in [HK98, Corollary 7.4]; see also Remark 5.9.

Let us describe in more detail the results in this paper. Section 2 is a brief overview on polygon spaces, where we give details for the symplectic reductions outlined above and for the moment polytope $\Xi:=\mu_{U(1)^{n}}^{-1}(G r(2, n))$. The polygon space $M(r)$ is a smooth Kähler manifold if and only if for any index set $I \subset\{1, \ldots, n\}$ the scalar quantity

$$
\varepsilon_{I}(r):=\sum_{I} r_{i}-\sum_{I^{c}} r_{i}
$$

never vanishes. When this is the case, the lengths vector $r$ is called generic.

In Section 3, we prove that, for $r$ generic, the piece-wise polynomial function for the symplectic volume of $M(r)$ is given by

$$
\operatorname{vol} M(r)=-\frac{(2 \pi)^{n-3}}{2(n-3) !} \sum_{I \text { long }}(-1)^{n-|I|} \varepsilon_{I}(r)^{n-3},
$$

where an index set $I$ is said to be long (or $r$-long) if and only if $\varepsilon_{I}(r)>0$. The symplectic volume of $M(r)$ was first computed by Takakura [Ta01] by means of a formula for the generating function of the intersection pairings of $M(r)$. Formula (1.1) was later obtained independently by $\mathrm{Vu}$ The Khoi 
in $[\mathbf{K h}]$. The equilateral polygon space $M(1, \ldots, 1)$ has some independent interest, and has been studied under several points of view, see for example $[\mathbf{B}, \mathbf{K i 9 2}]$. It is easy to see that the equilateral polygon space is smooth only for odd number of edges $n$. In this case, the symplectic volume of $M(1, \ldots, 1)$ has been computed by Kamiyama and Tezuka [KT], Takakura [Ta02] and by Martin [Ma]. In Section 3, we prove that Martin's techniques can be adapted to compute the volume of $M(r)$ (Theorem 3.4) for generic $r$ 's. We believe that this has some independent interests. The proof sets in the context of equivariant cohomology, where the surjectivity of the Kirwan map $k: H_{S O(3)}^{*}\left(\mathcal{S}_{r}\right) \rightarrow H^{*}(M(r))$ suggests that the calculation of the symplectic volume vol $M(r)$ can be done by looking at $\int_{M(r)} k(a)$ for a suitably chosen equivariant form $a \in H_{S O(3)}^{*}\left(\mathcal{S}_{r}\right)$ (i.e., such that $k$ maps $a$ onto the top power of the symplectic reduced form $\omega_{r}$ on $\left.M(r)\right)$. This is the natural setting for beautiful results, known as Localization Theorems, that enable one to localize the computation of the integral above at data associated to the fixed point set. Formula (1.1) is then an application of Martin's Localization Theorem (cf. [Ma] and Theorem 3.1 in here).

In Section 4, we deal with describing the diffeotype of $M(r)$ when $r$ crosses a wall in $\Xi$. It is well-known that for $r^{0}$ and $r^{1}$ on either side of a wall, the associated symplectic reductions $M\left(r^{0}\right)$ and $M\left(r^{1}\right)$ are related by a birational map that is the composite of a blow-up followed by a blow-down. This holds in greater generality, as proven in $[\mathbf{G S 8 9}, \mathbf{B P}]$. For polygon spaces we can characterize the submanifolds blown up and blown down as lower-dimensional polygon spaces; cf. Theorem 4.1. The moment polytope $\Xi$, first studied in [HK97], is the hypersimplex $\{r \in$ $\mathbb{R}_{+}^{n} \mid 0 \leq 2 r_{i} \leq 1$ and $\left.\sum_{i=1}^{n} r_{i}=1\right\}$. The regions $\Delta^{i}$ of regular values in $\Xi$ (called chambers) are separated by walls, which are the connected components of the image via $\mu_{U(1)^{n}}$ of the fixed points set $\operatorname{Gr}(2, n)^{H}$ for subgroups $H \subset U(1)^{n}$. It is not difficult to see that it is enough to consider the circles $\left\{\operatorname{diag}\left(e^{i \theta} \chi_{I}(1), \ldots, e^{i \theta} \chi_{I}(n)\right)\right\} \subset U(1)^{n}$ where, for any index set $I \subset\{1, \ldots, n\}$ index set, $\chi_{I}(i)=1$ if $i \in I$ and $\chi_{I}(j)=0$ if $j \in I^{c}$. It follows (see Section 2 and $[\mathbf{H K 9 7}]$ ) that the walls in $\Xi$ have equation

$$
\sum_{I} r_{i}-\sum_{I^{c}} r_{i}=0
$$

for some $I \subset\{1, \ldots, n\}$. The polygon space $M(r)$ is a smooth $(n-3)$ dimensional symplectic manifold if and only if equation (1.2) is never satisfied for any index set $I$. Consider

$$
\varepsilon_{I_{p}}(r)=\sum_{I_{p}} r_{i}-\sum_{I_{q}} r_{i}
$$

where the index set $I_{p}=\left\{i_{1}, \ldots i_{p}\right\}$ has cardinality $p$ and $I_{q}=\left\{j_{1}, \ldots j_{q}\right\}$ is its complement (hence $q:=n-p$ ). Let $W_{I_{p}}$ denote the data of the wall 


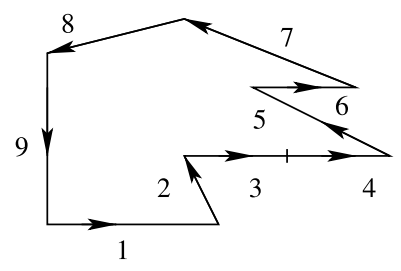

Figure 1. A polygon in $M_{I_{4}}(r)$ with $I_{4}=\{1,3,4,6\} \subset\{1, \ldots, 9\}$.

of equation $\varepsilon_{I_{p}}(r)=0$ together with the wall-crossing direction from the chamber where $\varepsilon_{I_{p}}(r)>0$ to the one where $\varepsilon_{I_{q}}(r)>0$. Throughout this paper we will only consider single wall-crossings, i.e., we assume that the wall-crossing point $r^{c}$ is not on a intersection of walls. This is not restrictive, since any non-single wall-crossing can be decomposed in a finite number of single wall-crossings. Let $r^{c} \in W_{I_{p}}$ be the wall-crossing point, i.e., $\varepsilon_{I_{p}}\left(r^{c}\right)=$ 0 . It follows that $\mu^{-1}(0)$ contains the $S O(3)$-orbit of the polygon $P^{c}=$ $\left(e_{1}^{c}, \ldots, e_{n}^{c}\right)$ with

$$
e_{i}^{c}= \begin{cases}\left(r_{i}, 0,0\right) & \text { if } i \in I_{p} \\ -\left(r_{i}, 0,0\right) & \text { if } i \in I_{q}\end{cases}
$$

The polygon $P^{c}$ lies completely on a line, the $x$-axis, and therefore it is fixed by the circle $S^{1}$ of rotations around it. This originates a singularity of conic type in the quotient $M\left(r^{c}\right)$. In Section 4, we analyze this singularity using the description of $M(r)$ as the symplectic quotient of $G r(2, n)$ by $U(1)^{n}$. In particular, we first perform reduction on $\operatorname{Gr}(2, n)$ by a complement $H$ of the circle $S^{1} \subseteq U(1)^{n} / U(1)$ associated to the wall. The residual $S^{1}$-action on $\operatorname{Gr}(2, n) /\left\{r_{2}, \ldots, r_{n-1}\right\} H$ is still Hamiltonian with moment map $\mu_{S^{1}}$. The wall-crossing problem for polygon spaces gets then reduced to studying the changes in the quotient

$$
\left(G r(2, n) / /\left\{r_{2}, \ldots, r_{n-1}\right\} H\right) / / r S^{1}
$$

when $r$ goes through a critical value of $\mu_{S^{1}}$. This provides us with two blowdown maps $\beta_{-}: M\left(r^{0}\right) \rightarrow M\left(r^{c}\right)$ and $\beta_{+}: M\left(r^{1}\right) \rightarrow M\left(r^{c}\right)$, where $r^{0}$ and $r^{1}$ are regular values respectively before and after the wall-crossing as above. To give an explicit description of the two maps $\beta_{-}$and $\beta_{+}$let us introduce some notation. Consider the submanifold $M_{I_{p}}(r) \subset M(r)$ of polygons such that the edges $e_{i}$, for $i \in I_{p}$, are parallel and point in the same direction as in Figure 1.

Note that $M_{I_{p}}(r)$ is naturally isomorphic to the moduli space $M\left(r_{I_{p}}\right)$ of $(p+1)$-gons with lengths vector $r_{I_{p}}:=\left(\sum_{i \in I_{p}} r_{i}, r_{j_{1}}, \ldots, r_{j_{q}}\right)$. It follows that $M_{I_{p}}\left(r^{0}\right)$ is empty. In fact, the condition $\varepsilon_{I_{p}}\left(r^{0}\right)>0$ implies that $\{1\}$ is an $r_{I_{p}}$-long edge, and therefore the closing condition $\sum e_{i}=0$ is never 
satisfied for any $\vec{e} \in \mathcal{S}_{r_{I_{p}}}$. On the other hand, $M_{I_{q}}\left(r^{0}\right) \simeq M\left(r_{I_{q}}\right)$ is not empty and, as proven in Section 4 , is diffeomorphic to the projective space $\mathbb{C P}^{p-2}$. Similarly, the submanifold $M_{I_{q}}\left(r^{1}\right)$ is empty while $M_{I_{p}}\left(r^{1}\right)$ is the projective space $\mathbb{C P}^{q-2}$. In Section 4 , we prove that $\beta_{-}$maps $M\left(r^{0}\right) \backslash M_{I_{q}}\left(r^{0}\right)$ diffeomorphically onto $M\left(r^{c}\right) \backslash\left[P^{c}\right]$, and $M_{I_{q}}\left(r^{0}\right)$ gets blown down via $\beta_{-}$to $\left[P^{c}\right]$. Similarly, $\beta_{+}$blows down $M_{I_{p}}\left(r^{1}\right)$ to $\left[P^{c}\right]$ and maps $M\left(r^{1}\right) \backslash M_{I_{p}}\left(r^{1}\right)$ diffeomorphically onto $M\left(r^{c}\right) \backslash\left[P^{c}\right]$. The spaces $M_{I_{p}}\left(r^{1}\right)$ and $M_{I_{q}}\left(r^{0}\right)$ are different resolutions of the singularity corresponding to the degenerate polygon $\left[P^{c}\right]$ in $M\left(r^{c}\right)$, and both are dominated by the blow-up $\widetilde{M}$ of $M\left(r^{c}\right)$ at the singular point, with exceptional divisor $E \simeq \mathbb{C P}^{q-2} \times \mathbb{C P}^{p-2} \simeq M_{I_{p}}\left(r^{1}\right) \times M_{I_{q}}\left(r^{0}\right)$.

This wall-crossing analysis and the volume formula intertwine in Section 5, where we describe the cohomology ring $H^{*}(M(r), \mathbb{C})$. In Section 5.1, we recall some results due to Guillemin and Sternberg [GS95] on the cohomology ring of reduced spaces. Section 5.2 is the heart of our description of $H^{*}(M(r))$. In fact, if $r^{0}$ is in an external chamber of the moment polytope $\Xi$, then $M\left(r^{0}\right)$ is toric and the Chern classes $c_{1}, \ldots, c_{n}$ of the $n$ complex line bundles associated with $\mu_{U(1)^{n}}^{-1}\left(r^{0}\right) \rightarrow M\left(r^{0}\right)$ generate the cohomology ring $H^{*}\left(M\left(r^{0}\right)\right)$. We prove by wall-crossing arguments that this holds for any regular value $r$ in any chamber of the moment polytope $\Xi$. In fact, any internal chamber can be reached from an external one by a finite number of single wall-crossings. First we prove, as an application of our wall-crossing analysis, that crossing a wall $W_{I_{p}}$ changes the dimensions of the cohomology groups of degree $k$ for $k$ an even integer in the interval $[2 \min (p, q)-2,2 \max (p, q)-4]$. Precisely, if $M\left(r^{0}\right)$ and $M\left(r^{1}\right)$ are polygon spaces before and after crossing the wall $W_{I_{p}}$, then

$$
\operatorname{dim} H^{k}\left(M\left(r^{1}\right)\right)=\operatorname{dim} H^{k}\left(M\left(r^{0}\right)\right)+1, \quad 2 p-2 \leq k \leq 2 q-4(\text { case } q \geq p)
$$

for $k$ even, and $H^{k}\left(M\left(r^{1}\right)\right)=H^{k}\left(M\left(r^{0}\right)\right)$ for any other value of $k$. In particular

$$
H^{k}\left(M\left(r^{1}\right)\right)=H^{k}\left(M\left(r^{0}\right)\right)=0 \quad \forall k \text { odd }
$$

This result may also be obtained from the Poincaré polynomial formulae of Klyachko [Kl] or Hausmann-Knutson [HK98]. This increasing in the dimension of the "middle" cohomology groups can be explicitly described in terms of the submanifolds $M_{I_{p}}\left(r^{1}\right)$ and $M_{I_{q}}\left(r^{0}\right)$. Assume for simplicity that $q \geq p$, and let $P D\left(\left[M_{I_{p}}\left(r^{0}\right)\right]\right)$ be the Poincaré dual of $M_{I_{p}}\left(r^{0}\right)$. Then the cohomology class $P D\left(\left[M_{I_{p}}\left(r^{0}\right)\right]\right)$ living in $H^{2 p-2}\left(M\left(r^{0}\right)\right)$ is zero, since $M_{I_{p}}\left(r^{0}\right)$ is empty. On the other side, $M_{I_{p}}\left(r^{1}\right)$ is not empty and the class of its Poincaré dual $P D\left(\left[M_{I_{p}}\left(r^{1}\right)\right]\right)$ determines the increase in the dimension of $H^{2 p-2}\left(M\left(r^{1}\right)\right)$.

The increase in higher-dimensional cohomology groups is given by the cup product $P D\left(\left[M_{I_{p}}\left(r^{1}\right)\right]\right) \smile c_{1}^{\alpha}\left(\mathcal{N}^{1}\right)$ of $P D\left(\left[M_{I_{p}}\left(r^{1}\right)\right]\right)$ with the first Chern 
class $c_{1}\left(\mathcal{N}^{1}\right)$, where $\mathcal{N}^{1}$ is the normal bundle to $M_{I_{p}}\left(r^{1}\right)$, and with its powers $c_{1}^{\alpha}\left(\mathcal{N}^{1}\right)$ for $\alpha=0, \ldots, q-p$, as prescribed by the Decomposition Theorem (see $[\mathbf{B B D}]$ and also [CM05], and Theorem 5.6 in here):

$$
H^{*}\left(M\left(r^{1}\right)\right)=H^{*}\left(M\left(r^{0}\right)\right) \oplus \bigoplus_{\alpha=0}^{q-p} \mathbb{C}\left(P D\left(\left[M_{I_{p}}\left(r^{1}\right)\right]\right) \smile c_{1}^{\alpha}\left(\mathcal{N}^{1}\right)\right) .
$$

We prove that $P D\left(\left[M_{I_{p}}\left(r^{1}\right)\right]\right)$ and $c_{1}\left(\mathcal{N}^{1}\right)$ are linear combinations of the first Chern classes $c_{1}, \ldots, c_{n}$, (cf. Proposition 5.7). It follows that $H^{*}\left(M\left(r^{1}\right)\right)$ is generated by $c_{1}, \ldots, c_{n}$ if $H^{*}\left(M\left(r^{0}\right)\right)$ is as well. This is the case for $r^{0}$ in an external chamber and therefore, by crossing a finite number of walls, for $r^{0}$ in any chamber of the moment polytope $\Xi$.

Applying the Duistermaat-Heckman Theorem one can then describe the cohomology ring of $M(r)$ as follows (Theorem 5.8):

$$
H^{*}(M(r), \mathbb{C}) \simeq \mathbb{C}\left[x_{1}, \ldots, x_{n}\right] / \operatorname{Ann}(\operatorname{vol} M(r)),
$$

where a polynomial $Q\left(x_{1}, \ldots, x_{n}\right) \in \operatorname{Ann}(\operatorname{vol} M(r))$ if and only if

$$
Q\left(\frac{\partial}{\partial r_{1}}, \ldots, \frac{\partial}{\partial r_{n}}\right) \operatorname{vol} M(r)=0
$$

\section{The moduli space of polygons $M(r)$}

Let $S_{t}^{2}$ be the sphere in $\mathbb{R}^{3}$ of radius $t$ and center the origin. For $r=$ $\left(r_{1}, \ldots, r_{n}\right) \in \mathbb{R}_{+}^{n}$, the product $\mathcal{S}_{r}=\prod_{j=1}^{n} S_{r_{j}}^{2}$ of $n 2$-spheres is a smooth manifold. Let $p_{j}: \mathcal{S}_{r} \rightarrow S_{r_{j}}^{2}$ be the projection on the $j$ th factor and let $\omega_{j}$ be the volume form on the sphere $S_{r_{j}}^{2}$. Because the $\omega_{j}$ 's are closed and nondegenerate, the 2 -form $\omega=\sum_{j=1}^{n} \frac{1}{r_{j}} p_{j}^{*} \omega_{j}$ is closed and non-degenerate as well and defines a symplectic structure on $\mathcal{S}_{r}$. Note that the symplectic form $\omega$ can be written equivalently as $\sum_{j=1}^{n} r_{j} \pi_{j}^{*} \omega_{S^{2}}$ where $\pi_{j}$ is the composition of $p_{j}$ with the rescaling map $S_{r_{j}}^{2} \rightarrow S^{2}$, for details see [KM, Section $\mathbf{1}$ ].

The group $S O(3)$ acts diagonally on $\mathcal{S}_{r}$. Equivalently, identifying the sphere $S_{r_{j}}^{2}$ with a $S O(3)$-coadjoint orbit, the $S O(3)$-action on each sphere is the coadjoint one. The choice of an invariant inner product on the Lie algebra $\mathfrak{s o}(3)$ of $S O(3)$ induces an identification $\mathfrak{s o}(3)^{*} \simeq \mathbb{R}^{3}$ between the dual of $\mathfrak{s o}(3)$ and $\mathbb{R}^{3}$. On each sphere $S_{r_{j}}^{2}$, the moment map associated to the coadjoint action is the inclusion of $S_{r_{j}}^{2}$ in $\mathbb{R}^{3}$. By linearity, the diagonal action of $S O(3)$ on $\mathcal{S}_{r}$ has moment map

$$
\begin{aligned}
\mu: \mathcal{S}_{r} & \rightarrow \mathbb{R}^{3}, \\
\vec{e}=\left(e_{1}, \ldots, e_{n}\right) & \mapsto e_{1}+\cdots+e_{n} .
\end{aligned}
$$

The level set $\mu^{-1}(0):=\widetilde{M}(r)=\left\{\vec{e}=\left(e_{1}, \ldots, e_{n}\right) \in \mathcal{S}_{r} \mid \sum_{i=1}^{n} e_{i}=0\right\}$ is a submanifold of $\mathcal{S}_{r}$ because 0 is a regular value for $\mu$. 
A polygon in $\mathbb{R}^{3}$ is a closed piece-wise linear path in $\mathbb{R}^{3}$. Consider the piece-wise linear path such that the $j$ th step is given by the vector $e_{j}$. Such a path closes if and only if $\sum_{i=1}^{n} e_{i}=0$. Therefore, $\widetilde{M}(r)$ is the space of $n$-gons of fixed sides length $r_{1}, \ldots, r_{n}$. Its quotient

$$
M(r):=\widetilde{M}(r) / S O(3)=\mathcal{S}_{r} / / S O(3)
$$

is the space of $n$-gons of fixed sides length $r_{1}, \ldots, r_{n}$ modulo rigid motions, and is usually called polygon space. A polygon is called degenerate if it lies completely on a line.

The moduli space $M(r)$ is a smooth manifold if and only if the lengths vector $r$ is generic, i.e., for each $I \subset\{1, \ldots, n\}$, the quantity

$$
\varepsilon_{I}(r):=\sum_{i \in I} r_{i}-\sum_{i \in I^{c}} r_{i}
$$

is non-zero. Equivalently, if and only if in $M(r)$ there are no degenerate polygons. In fact, if there exists a polygon $P$ on a line (or an index set $I$ such that $\left.\varepsilon_{I}(r)=0\right)$ then its stabilizer is $S^{1}$ since the polygon $P$ is fixed by rotations around the axis it defines. Therefore the $S O(3)$-action on $\widetilde{M}(r)$ is not free and the quotient has singularities, which have been studied by Kapovich and Millson in $[\mathbf{K M}]$. Precisely, they proved that $M(r)$ is a complex analytic space with isolated singularities corresponding to the degenerate $n$-gons in $M(r)$, and these singularities are equivalent to homogeneous quadratic cones. Along the proof of the wall-crossing Theorem 4.1 in Section 4, we will provide an explicit description of the cone $C_{W}$ over the singularity. Note that, for $r$ generic, the polygon space $M(r)$ inherits a symplectic form by symplectic reduction, see for example $[\mathbf{A u}]$.

An alternative description of the moduli space $M(r)$ is given by Hausmann and Knutson in [HK97] which also resemble an earlier work of Gelfand and MacPherson $[\mathbf{G M}]$. With minor adaptations we provide an overview here. Let $U(1)^{n}$ be the maximal torus of diagonal matrices in the unitary group $U(n)$. The group $U(1)^{n} \times U(2)$ acts by conjugation on $\mathbb{C}^{n \times 2}$. The action is Hamiltonian and the polygon space $M(r)$ can then be realized as the symplectic quotient of $\mathbb{C}^{n \times 2}$ by $U(1)^{n} \times U(2)$; cf. [HK97]. One can perform reduction in stages. Consider first the $U(2)$-action with associated moment map

$$
\begin{aligned}
\mu_{U(2)}: \mathbb{C}^{n \times 2} & \rightarrow \mathfrak{u}(2)^{*}, \\
A & \mapsto-\frac{i}{2}\left(A^{*} A-I d\right),
\end{aligned}
$$


where $A^{*}$ is the conjugate transpose of $A$ and $I d$ is the identity matrix. The Stiefel manifold of orthonormal 2 frames in $\mathbb{C}^{n}$, defined as follows

$$
S t_{2, n}=\left\{\left(\begin{array}{cc}
a_{1} & b_{1} \\
\vdots & \vdots \\
a_{n} & b_{n}
\end{array}\right) \in \mathbb{C}^{n \times 2}: \sum_{i=1}^{n}\left|a_{i}\right|^{2}=1, \sum_{i=1}^{n}\left|b_{i}\right|^{2}=1, \sum_{i=1}^{n} a_{i} \bar{b}_{i}=0\right\}
$$

can be realized as the zero level set $\mu_{U(2)}^{-1}(0)$. Let $G r(2, n)$ be the Grassmannian of 2-planes in $\mathbb{C}^{n}$. The map

$$
p: S t_{2, n} \rightarrow \operatorname{Gr}(2, n)
$$

that takes an element $(a, b) \in S t_{2, n}$ into the plane generated by the column vectors $a$ and $b$ is actually the projection of $S t_{2, n}$ on to the orbit space $S t_{2, n} / U(2)$. This realizes the Grassmannian $G r(2, n)$ as the symplectic quotient $\mathbb{C}^{n \times 2} / / U(2)$. The projection $p$ is $U(n)$-equivariant and thus the $U(n)$ action descends to an action on the quotient $\operatorname{Gr}(2, n)$. The action of the maximal torus $U(1)^{n}$ on $G r(2, n)$ is Hamiltonian with associated moment map $\mu_{U(1)^{n}}: \operatorname{Gr}(2, n) \rightarrow \mathbb{R}^{n}$ such that, if $\Pi=\langle a, b\rangle$ is the plane generated by $a, b \in \mathbb{C}^{n}$, then

$$
\mu_{U(1)^{n}}(\Pi)=\frac{1}{2}\left(\left|a_{1}\right|^{2}+\left|b_{1}\right|^{2}, \ldots,\left|a_{n}\right|^{2}+\left|b_{n}\right|^{2}\right) .
$$

The image of the moment map $\mu_{U(1)^{n}}(G r(2, n))$ is the hypersimplex $\Xi$

$$
\mu_{U(1)^{n}}(G r(2, n))=\Xi=\left\{\left(r_{1}, \ldots, r_{n}\right) \in \mathbb{R}^{n} \mid 0 \leq 2 r_{i} \leq 1, \quad \sum_{i=1}^{n} r_{i}=1\right\}
$$

and the set of critical values of $\mu_{U(1)^{n}}$ consists of those points $\left(r_{1}, \ldots, r_{n}\right) \in \Xi$ satisfying one of the following conditions

(a) one of the $r_{i}$ 's vanishes or is equal to $1 / 2$;

(b) $\exists I$ such that $\varepsilon_{I}(r)=0$ with $|I|$ and $\left|I^{c}\right|$ at least two.

Remark 2.1. Note that points satisfying (a) constitute the boundary of $\Xi$, while points satisfying condition (b) are the inner walls of $\Xi$. Moreover, condition (a) is equivalent to the following

$\left(\mathrm{a}^{\prime}\right) \exists I$ such that $\varepsilon_{I}(r)=0$ with $|I|=1$ or $|I|=n-1$

Therefore, a wall in $\Xi$ has equation

$$
\varepsilon_{I}(r)=-\varepsilon_{I^{c}}(r)=0
$$

for some index subset $I \subset\{1, \ldots, n\}$. Denote by $W_{I}$ or $W_{I^{c}}$ the wall of equation (2.1). (In Section 4, the choice of either $W_{I}$ or $W_{I^{c}}$ will encode the wall crossing direction.) The walls separates the regions $\Delta^{i}$ of regular values, called chambers, for which $\varepsilon_{I}(r) \neq 0$ for any $I \subset\{1, \ldots, n\}$. An index set $I$ is said to be short if $\varepsilon_{I}(r)<0$, and long if its complement is short. Geometrically, an index set $I$ is short if the polygon space $M(r)$ 
contains configurations $\left[e_{1}, \ldots, e_{n}\right]$ where the edges $e_{i}$, for $i \in I$, are all positive proportional to each other. For example, a polygon as in figure 1 exists in $M(r)$ if and only if the index set $I_{4}=\{1,3,4,6\}$ is short.

Since for any regular value either $I$ or $I^{c}$ is short, and this is consistent within the chamber $\Delta^{i}$ containing $r$, it follows that a chamber $\Delta^{i}$ is uniquely determined by the collection of short sets

$$
\mathcal{S}\left(\Delta^{i}\right):=\left\{I \subset\{1, \ldots, n\} \mid I \text { is short for any } r \in \Delta^{i}\right\} .
$$

Given $I \in \mathcal{S}\left(\Delta^{i}\right)$, the wall $W_{I}$ is in the closure of $\Delta^{i}$ if and only if $I$ is maximal (with respect to the inclusion) in $\mathcal{S}\left(\Delta^{i}\right)$. A chamber $\Delta^{i}$ is external if its closure contains an outer wall. Equivalently, this means that there exists a cardinality- 1 set $\{j\} \in \mathcal{S}\left(\Delta^{i}\right)$ which is not contained in any other short set.

Under a canonical diffeomorphism between $M(r)$ and $M(\lambda r)$ the symplectic forms are proportional $\left(\omega_{\lambda r}=\lambda \omega_{r}\right)$. Hence, the condition that fixes the perimeter $\sum_{i=1}^{n} r_{i}=1$ is not restrictive and allows one to work with the compact polytope $\Xi$ rather than with the positive octant $\mathbb{R}_{+}^{n}$, whose chambers of regular values are cones. The particular choice $\sum_{i=1}^{n} r_{i}=1$ descends from the moment map $\mu_{U(2)}$ (or equivalently from considering orthonormal frames in $\left.S t_{2, n}\right)$.

Proposition 2.2. (Hausmann-Knutson [HK97]) For generic $r \in \Xi$, the polygon space $M(r)$ is the symplectic reduction relative to the $U(1)^{n}$-action on the Grassmaniann $\operatorname{Gr}(2, n)$ at the level set $r$, i.e.,

$$
M(r) \simeq U(1)^{n} \backslash \mu_{U(1)^{n}}^{-1}(r)=G r(2, n) / /{ }_{r} U(1)^{n} .
$$

Note that one recovers the previous description of $M(r)$ as the symplectic quotient $\mathcal{S}_{r} / / S O(3)$ performing the reduction of $\mathbb{C}^{n \times 2}$ by $U(1)^{n} \times U(2)$ in the opposite order. In fact, let $\tilde{\mu}_{U(1)^{n}}: \mathbb{C}^{n \times 2} \rightarrow \mathbb{R}^{n}$ be the moment map for the $U(1)^{n}$ action on $\mathbb{C}^{n \times 2}$. Clearly

$$
\tilde{\mu}_{U(1)^{n}}(a, b)=\frac{1}{2}\left(\left|a_{1}\right|^{2}+\left|b_{1}\right|^{2}, \ldots,\left|a_{n}\right|^{2}+\left|b_{n}\right|^{2}\right)
$$

and

$$
\tilde{\mu}_{U(1)^{n}}^{-1}(r) \simeq \prod_{j=1}^{n} S_{\sqrt{2 r_{j}}}^{3} .
$$

The torus $U(1)^{n}$ acts diagonally on $\prod_{j=1}^{n} S_{\sqrt{2 r_{j}}}^{3}$ and the projection map

$$
\tilde{\mu}_{U(1)^{n}}^{-1}(r) \rightarrow \mathbb{C}^{n \times 2} / / r U(1)^{n}
$$


is just the Hopf map

$$
\begin{aligned}
H^{n}: \prod_{j} S_{\sqrt{2 r_{j}}}^{3} & \rightarrow \prod_{j} S_{r_{j}}^{2}, \\
(a, b) & \mapsto\left(H\left(a_{1}, b_{1}\right), \ldots, H\left(a_{n}, b_{n}\right)\right),
\end{aligned}
$$

where

$$
H\left(a_{i}, b_{i}\right)=\left(\frac{\left|a_{i}\right|^{2}-\left|b_{i}\right|^{2}}{2}, \operatorname{Re}\left(\bar{a}_{i} b_{i}\right), \operatorname{Im}\left(\bar{a}_{i} b_{i}\right)\right)
$$

and $\operatorname{Re}\left(\bar{a}_{i} b_{i}\right)$ and $\operatorname{Im}\left(\bar{a}_{i} b_{i}\right)$ are the real and imaginary part of $\bar{a}_{i} b_{i} \in \mathbb{C}$. Note that on each sphere the map $H^{n}$ is obtained from the quaternionic Hopf map

$$
\tilde{H}\left(a_{\ell}, b_{\ell}\right)=i\left[\left(\left|a_{\ell}\right|^{2}-\left|b_{\ell}\right|^{2}\right)+2 \bar{a}_{\ell} b_{\ell} j\right] .
$$

The residual $U(2) / U(1) \simeq S O(3)$ action is then the one described above.

Since these two descriptions of $M(r)$ obtained by performing reduction in stages in different order will play a central role along the paper, we find it convenient to explore here the relation between the two. Denote by $p^{-1}\left(\mu_{U(1)^{n}}^{-1}(r)\right)$ the preimage in $S t_{2, n}$ of the $r$-level set in $G r(2, n)$. Then $p^{-1}\left(\mu_{U(1)^{n}}^{-1}(r)\right)$ is the set of $(a, b) \in S t_{2, n}$ such that each row has norm $2 r_{i}$, i.e.,

$$
p^{-1}\left(\mu_{U(1)^{n}}^{-1}(r)\right)=\left\{(a, b) \in S t_{2, n}:\left|a_{i}\right|^{2}+\left|b_{i}\right|^{2}=2 r_{i} \quad \forall i=1, \ldots n\right\} .
$$

This naturally defines the inclusion map

$$
\imath: p^{-1}\left(\mu_{U(1)^{n}}^{-1}(r)\right) \hookrightarrow \tilde{\mu}_{U(1)^{n}}^{-1}(r) \simeq \prod_{j} S_{\sqrt{2 r_{j}}}^{3} .
$$

It follows that

$$
H^{n}\left(\imath\left(p^{-1}\left(\mu_{U(1)^{n}}^{-1}(r)\right)\right)\right)=\mu_{S O(3)}^{-1}(0)=\left\{\left(e_{1}, \ldots, e_{n}\right) \in \prod_{j} S_{r_{j}}^{2} \mid \sum_{i} e_{i}=0\right\} .
$$

The fact that the vectors $e_{i}:=H\left(a_{i}, b_{i}\right)$ sum up to 0 follows from the conditions for $(a, b) \in S t_{2, n}$ :

$$
H\left(a_{i}, b_{i}\right)=\left(\sum_{i=1}^{n} \frac{\left|a_{i}\right|^{2}-\left|b_{i}\right|^{2}}{2}, \sum_{i=1}^{n} \operatorname{Re}\left(\bar{a}_{i} b_{i}\right), \sum_{i=1}^{n} \operatorname{Im}\left(\bar{a}_{i} b_{i}\right)\right)=0 .
$$


Thus, the following diagram embodies the rich geometric structure of $M(r)$ : (2.3)

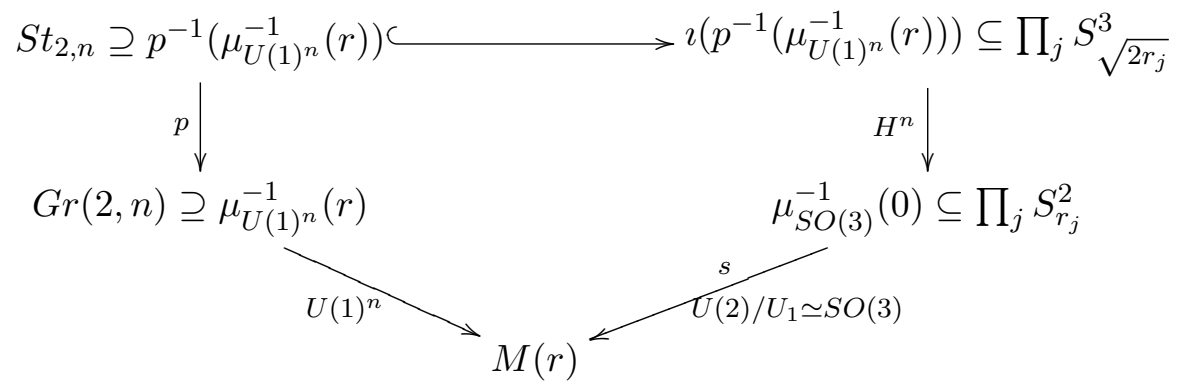

Remark 2.3. In $[\mathbf{K M}]$ Kapovich and Millson define the so-called bending flows on the polygon space $M(r)$. As proved in [HK97], these form the residual torus action from the Gelfand-Cetlin system on the Grassmannian $\operatorname{Gr}(2, n)$. The bending flows define a toric action on the polygon space $M(r)$ if and only if it is possible to choose a system of $n-3$ nonintersecting and nowhere vanishing diagonals. For $n=4,5,6$, Hausmann and Knutson [HK97, Section 6] determine explicit combinatorial conditions depending on $r \in \mathbb{R}_{+}^{n}$ for the bending action on $M(r)$ to be toric; see also [M]. In particular, for $n=5$, in [HK00], Hausmann and Knutson prove that $M(1,1,1,1,1)$ is not toric. In fact, it has Riemann-Roch number 6 and Euler characteristic 7 . Still, for small $\varepsilon$, the length vector $(1+\varepsilon, 1,1,1,1+\varepsilon)$ is in the same chamber as $(1,1,1,1,1)$ and the bending flows define a toric action on the polygon space $M(1+\varepsilon, 1,1,1,1+\varepsilon)$; cf. [HK97, HK00]. Consequently in the same chamber, we can obtain both toric and non-toric manifolds, i.e., being toric is not an invariant of the chamber.

\section{The symplectic volume of $M(r)$}

The goal of this of this section is to prove an explicit formula for the volume of polygon spaces. The volume of $M(r)$ had been already computed $[\mathbf{T a 0 1}, \mathbf{K h}]$, still we believe that the proof we give via Martin's localization Theorem has some independent interest. Moreover, the volume formula Theorem 3.4 has a central role for our description of the cohomology ring $H^{*}(M(r), \mathbb{C})$ in Theorem 5.8.

3.1. Martin's results. In this section, we give some basic definitions and results in equivariant cohomology. On this topic, there is a rich literature, in particular we refer to the survey papers $[\mathbf{A B}]$ and $[\mathbf{D u}]$, and also the book $[\mathbf{K i}]$.

Let $G$ be a compact Lie group acting on a smooth manifold $M$ in a Hamiltonian way, with moment map $\mu: M \rightarrow \mathfrak{g}^{*}$. The equivariant cohomo$\log y$ of $M$ is defined to be the ordinary cohomology of $M_{G}:=E G \times_{G} M$, 
where $E G$ is the total space of the universal bundle $E G \rightarrow B G, B G$ being the classifying space of the group $G$.

Let $\xi \in\left(\mathfrak{g}^{*}\right)^{G}$ be a regular value for the moment map $\mu$, fixed by the co-adjoint $G$-action. Assume also that $G$ acts freely on $\mu^{-1}(\xi)$, so that the orbit space $\mu^{-1}(\xi) / G:=M / / \xi$ is a manifold.

In $[\mathbf{K i}]$, Kirwan proved that there exists an epimorphism

$$
k: H_{G}^{*}(M) \rightarrow H^{*}\left(M /{ }_{\xi} G\right),
$$

which is known as the Kirwan map.

The surjectivity of the Kirwan map rises the hope that a good deal of information about the cohomology ring $H^{*}(M / / \xi G)$ of a reduced space can be computed from the equivariant cohomology $H_{G}^{*}(M)$ of $M$. The extra information encoded by the equivariant cohomology turns out to be related to the orbit structure of the $G$-action, and in this sense equivariant cohomology is the natural setting for results, known as localization Theorems, which enables many computation to be reduced to the fixed point set of the $G$-action.

Our proof of the volume formula (Theorem 3.4) for the moduli space of polygons is based on a localization Theorem due to Martin [Ma]. A similar result has been proven independently by Guillemin and Kalkman $[\mathbf{G K}]$. In $[\mathbf{M a}]$ it is calculated, as an example, the symplectic volume of the moduli space $M(1, \ldots, 1)$ of polygons with an odd number of edges all of length 1 . In Section 3.2, we prove that, mutata mutandis, Martin's techniques hold for any generic $r \in \mathbb{R}_{+}^{n}$.

Assume now that $M$ is symplectic. Endow $M$ with a Hamiltonian action of a torus $T$ with associated moment map $\mu: X \rightarrow \mathfrak{t}^{*}$. Let $p_{0}$ and $p_{1}$ be two regular values of the moment map $\mu$. A transverse path $Z$ is a onedimensional submanifold $Z \subset \mathfrak{t}^{*}$ with boundary $\left\{p_{0}, p_{1}\right\}$ such that $Z$ is transverse to $\mu$. A wall in $\mathfrak{t}^{*}$ is defined to be a connected component of $\mu\left(M^{H}\right)$ where $M^{H}$ is the fixed point set for some oriented subgroup $H \simeq S^{1}$ of $T$.

Orient $H$ as follows: first orient $Z$ from $p_{0}$ to $p_{1}$. Each positive tangent vector field in $T_{q} Z$, thought as an element of $\mathfrak{t}^{*}$, defines a functional on $\mathfrak{t}$ which restricts to a non-zero functional on $\mathfrak{h}:=\operatorname{Lie}(H)$. The orientation of $H$ is defined to be the positive one with respect to this functional.

Theorem 3.1 (Localization Theorem [Ma]). Let $p_{0}$ and $p_{1}$ be regular values of the moment map $\mu$ joined by a transverse path $Z$ having a single wall-crossing at $q$ and let $H \simeq S^{1}$ be the oriented subgroup associated to the wall-crossing from $p_{0}$ to $p_{1}$. There exists a map

$$
\lambda_{H}: H_{T}^{*}(M) \rightarrow H_{T / H}^{*}\left(M^{H}\right),
$$


called localization map, such that, for any $a \in H_{T}^{*}(X)$,

$$
\int_{M / p_{0} T} k_{0}(a)-\int_{M / p_{1} T} k_{1}(a)=\int_{M^{H} / / q^{T}} k_{q}\left(\lambda_{H}\left(a_{\left.\right|_{M} H}\right)\right)
$$

where the maps $k_{i}: H_{T}^{*}(M) \rightarrow H^{*}\left(M / p_{i} T\right)$ are the Kirwan maps, $M^{H} \|_{q} T$ is the symplectic quotient of $\mu_{\left.\right|_{M} H}^{-1}(q) \cap M^{H}$ by the quotient subgroup $T / H$ and $k_{q}: H_{T / H}^{*}\left(M^{H}\right) \rightarrow H^{*}\left(M^{H} /{ }_{q} T\right)$ is the associated Kirwan map.

It is possible to describe the localization map $\lambda_{H}$ in terms of equivariant characteristic classes. As pointed out in $[\mathbf{A B}]$, the functorial nature of the construction that to $M$ associates $M_{G}$ enables one to define equivariant correspondents of the concepts of ordinary cohomology in a natural way. In particular, if $V$ is a vector bundle over $M$, then any action of $G$ on $V$ lifting the action on $M$ can be used to define a vector bundle $V_{G}=E G \times{ }_{G} V$ over $M_{G}$ that extends the bundle $V \rightarrow M$. Thus, for example, the first Chern class of $V_{G}, c_{1}\left(V_{G}\right)$, naturally lies in $H^{*}\left(M_{G}\right)=: H_{G}^{*}(M)$ and is called the equivariant first Chern class of $V$, denoted by $c_{1}^{G}(V)$. All other equivariant characteristic classes are defined in a similar way. (See also [Ma], Appendix B.)

As before, $H \simeq S^{1}$ is the subgroup of $T$ associated to the wall-crossing we are examining. Let $T^{\prime} \subset T$ be a complement of $H$, i.e., $T=T^{\prime} \times H$. This defines an isomorphism $H_{T}^{*}\left(M^{H}\right) \cong H_{T^{\prime}}^{*}\left(M^{H}\right) \otimes H_{H}^{*}\left(M^{H}\right)$. Note that $H_{H}^{*}\left(M^{H}\right) \cong H^{*}(B H)$ (it is enough to remember that $H_{H}^{*}\left(M^{H}\right)$ is defined to be the ordinary cohomology ring $H^{*}\left(E H \times_{H} M^{H}\right)$ and to note that $H$ acts trivially on its fixed point set $\left.M^{H}\right)$. Therefore

$$
H_{T}^{*}\left(M^{H}\right) \cong H_{T^{\prime}}^{*}\left(M^{H}\right) \otimes H^{*}(B H) .
$$

It follows that the restriction to $M^{H}$ of any class $a \in H_{T}^{*}(M)$ decomposes as $a_{\left.\right|_{M^{H}}}=\sum_{i \geq 0} a_{i} \otimes u^{i}$ where $u$ is the positive generator of $H^{*}(B H)$ and the $a_{i}$ are elements in $H_{T^{\prime}}^{*}\left(M^{H}\right)$.

Proposition 3.2 ( [Ma]). With the notation above,

$$
\lambda_{H}(a)=k\left(M_{i}^{H}\right) \sum_{i \geq 0} a_{i} \smile s_{i-\rho+1}^{w},
$$

where $k\left(M_{i}^{H}\right)$ is the greatest common divisor of the weights of the H-action on the fibers of the normal bundle $\nu M_{i}^{H} \rightarrow M_{i}^{H}, s_{j}^{w}$ denotes the $j$ th $T^{\prime}$ equivariant Segre class of $\left(\nu M^{H}, H\right)$ and $\rho$ is the function (constant on the connected components of $\left.M^{H}\right)$ such that $2 \rho=\operatorname{rank}\left(\nu M^{H}\right)$.

The next result relate integration over the symplectic quotients respectively by a non abelian group $G$ and by a maximal torus in $G$.

Let $G$ be a connected compact Lie group, which acts on the smooth manifold $M$ in a Hamiltonian way, with associated moment map $\mu_{G}$. Let 
$T$ be a maximal subtorus in $G$. The restriction of the $G$-action defines a Hamiltonian $T$-action on $M$ (with associated moment map $\mu_{T}$ ). There is a natural restriction map $r_{T}^{G}: H_{G}^{*}(M) \rightarrow H_{T}^{*}(M)$ between the equivariant (with respect to $G$ and $T$ ) cohomology rings. To fix the notation, $\mathbb{C}_{(w)}^{m}$ denotes the complex space $\mathbb{C}^{m}$ endowed of the $S^{1}$-action with weight $w$ and $\mathbb{C}_{(w)}:=M \times \mathbb{C}_{w}$ is the total space of an equivariant line bundle over $M$.

Theorem 3.3 (Equivariant integration formula [Ma2]). For all $a \in$ $H_{G}^{*}(M)$,

$$
\int_{M / / G} k_{G}(a)=\frac{1}{|W|} \int_{M / / T} k_{T}\left(r_{T}^{G}(a) \smile \prod_{\alpha \in \Delta} c_{1}^{T}\left(\underline{\mathbb{C}}_{\alpha}\right)\right),
$$

where $|W|$ is the order of the Weyl group of $G$ and $\Delta$ is the set of roots of $G$.

\subsection{The volume theorem.}

Theorem 3.4. For generic $r \in \mathbb{R}_{+}^{n}$,

$$
\operatorname{vol} M(r)=-\frac{(2 \pi)^{n-3}}{2(n-3) !} \sum_{\text {Ilong }}(-1)^{n-|I|} \varepsilon_{I}(r)^{n-3}
$$

where $I \subset\{1, \ldots, n\}$ is long if and only if $\varepsilon_{I}(r)=\sum_{i \in I} r_{i}-\sum_{i \in I^{c}} r_{i}>0$.

Proof. The first step in the proof is to apply Theorem 3.3 and write the volume of $M(r)$ as

$$
\operatorname{vol} M(r)=\frac{1}{2} \int_{\mathcal{S}_{r} / / S^{1}} k_{S^{1}}\left(r_{S^{1}}^{S O(3)}(a) \smile c_{1}^{S^{1}}\left(\underline{\mathbb{C}}_{(1)}\right) \smile c_{1}^{S^{1}}\left(\underline{\mathbb{C}}_{(-1)}\right)\right),
$$

where $a \in H_{S O(3)}^{*}\left(\mathcal{S}_{r}\right)$ is such that $k_{S O(3)}(a)$ is the volume form on $\mathcal{S}_{r} / / S O(3)=M(r)$ and $S^{1}$ is a (arbitrarily chosen) maximal subtorus of $S O(3)$. (We have already entered in the formula that the Weyl group of $S O(3)$ is $\mathbb{Z} / 2 \mathbb{Z}$ and that the set of roots of $S O(3)$ is $\{ \pm 1\}$.)

The second step is to apply the localization Theorem 3.1 to localize the calculation of the integral above to data associated to the fixed points set of the $S^{1}$-action.

Remember that the symplectic structure on $\mathcal{S}_{r}$ is defined by the 2 -form $\omega=\sum_{j=1}^{n} \frac{1}{r_{j}} p_{j}^{*} \omega_{j}$, where $p_{j}: \mathcal{S}_{r} \rightarrow S_{r_{j}}^{2}$ is the natural projection on the $j$ th factor and $\omega_{j}$ is the volume form on the sphere $S_{r_{j}}^{2}$. It is a calculation to check that, if $\alpha$ is the volume form on the unit sphere and $\omega_{\mathrm{FS}}$ is the Fubini-Study form on $\mathbb{C P}^{1} \simeq S^{2}$, then $\omega_{j}=r_{j} \alpha=2 r_{j} \omega_{\mathrm{FS}}$.

On each sphere consider the line bundle $\mathcal{O}\left(2 r_{j}\right) \rightarrow S_{j}^{2}$. The tensor product of their pullbacks $p_{j}^{*} \mathcal{O}\left(2 r_{j}\right)$ defines on $\mathcal{S}_{r}$ the line bundle $\mathcal{L}:=\mathcal{O}\left(2 r_{1}\right) \otimes \cdots \otimes$ $\mathcal{O}\left(2 r_{n}\right)$ (known in literature as the prequantum line bundle of $\mathcal{S}_{r}$ ). Observe 
that $\omega_{\mathrm{FS}}$ is the first Chern class of $\mathcal{O}(1)$, precisely $\left[\frac{\omega_{\mathrm{FS}}}{2 \pi}\right]=c_{1}(\mathcal{O}(1))$. It follows by the definition of the symplectic form $\omega$ on $\mathcal{S}_{r}$ that

$$
\left[\frac{\omega}{2 \pi}\right]=c_{1}\left(\mathcal{O}\left(2 r_{1}\right) \otimes \cdots \otimes \mathcal{O}\left(2 r_{n}\right)\right)=c_{1}(\mathcal{L}) .
$$

The construction above is well defined just for integral $r_{1}, \ldots, r_{n}$, so let us restrict to the case $r \in \mathbb{Z}_{+}^{n}$ and prove the stated result for the volume of $M(r)$. Then, for each $\lambda \in \mathbb{R}^{+}$, we get the volume of $M(\lambda r)$ by rescaling. Indeed, vol $M(\lambda r)=(\lambda)^{n-3}(\operatorname{vol} M(r))$, thus the formula holds also for rational $r_{i}$. Finally, by density, the result extends to $r \in \mathbb{R}_{+}^{n}$.

Let $a$ be the $(n-3)$ th power of the first equivariant Chern class $c_{1}^{S O(3)}(\mathcal{L})$ of the prequantum line bundle $\mathcal{L}$ (normalized by a factor $\frac{(2 \pi)^{n-3}}{(n-3) !}$ ). Then its image $k(a)$ through the Kirwan map $k: H_{S O(3)}^{*}\left(\mathcal{S}_{r}\right) \rightarrow H^{*}\left(\mathcal{S}_{r} / / S O(3)\right)$ is the volume form on $M(r)$ :

$$
\operatorname{vol} M(r)=\frac{(2 \pi)^{n-3}}{(n-3) !} \int_{M(r)} k\left(c_{1}^{S O(3)}(\mathcal{L})^{n-3}\right) .
$$

We now apply the equivariant integration formula (Theorem 3.3). The restriction $r_{S^{1}}^{S O(3)} \operatorname{maps} c_{1}^{S O(3)}(\mathcal{L})^{n-3}$ to $c_{1}^{S^{1}}(\mathcal{L})^{n-3}$, thus

$$
\operatorname{vol} M(r)=\frac{1}{2} \frac{(2 \pi)^{n-3}}{(n-3) !} \int_{\mathcal{S}_{r} / / S^{1}} k_{S^{1}}\left(c_{1}^{S^{1}}(\mathcal{L})^{n-3} \smile c_{1}^{S^{1}}\left(\underline{\mathbb{C}}_{(1)}\right) \smile c_{1}^{S^{1}}\left(\underline{\mathbb{C}}_{(-1)}\right)\right)
$$

and the first step is done.

In order to apply the localization Theorem 3.1 we make an explicit choice of a maximal subtorus $S^{1} \subset S O(3)$ : let $S^{1}$ be the subgroup that acts on each sphere by rotation along the $z$-axis. This action is Hamiltonian with moment map the height function

$$
\begin{aligned}
\mu: S_{r_{j}}^{2} & \rightarrow \mathfrak{s}^{1} \simeq \mathbb{R}, \\
e_{j}=\left(x_{j}, y_{j}, z_{j}\right) & \mapsto h t\left(e_{j}\right)=z_{j} .
\end{aligned}
$$

Note that the fixed points of this action are the north pole $N_{j}$ and the south pole $S_{j}$ and the image $\mu\left(S_{r_{j}}^{2}\right)$ is the segment $\left[\mu\left(S_{j}\right), \mu\left(N_{j}\right)\right]=\left[-r_{j}, r_{j}\right]$ (in agreement with the convexity Theorem).

These observations extend easily to the product manifold $\mathcal{S}_{r}$ : consider on $\mathcal{S}_{r}$ the circle action by rotation around the $z$-axis of each sphere. This action is Hamiltonian and, by linearity, has moment map the sum of the heights, i.e., $\mu\left(e_{1}, \ldots, e_{n}\right)=\sum_{j=1}^{n} z_{j}$.

A point $\left(e_{1}, \ldots, e_{n}\right)$ is fixed by this action if and only if $e_{j} \in\left\{N_{j}, S_{j}\right\}$ for each $j \in\{1, \ldots, n\}$, and these points are isolated. For these points we introduce a more handy notation: let $I$ be any subset of $\{1, \ldots, n\}$. We define $f_{I}$ to be the point $\left(e_{1}, \ldots, e_{n}\right) \in \mathcal{S}_{r}$ such that $e_{j}$ is a north pole if $j \in I$ and 
a south pole if $j \in I^{c}$. Thus, all the fixed points are an $f_{I}$ for some index set $I$ and

$$
\mu\left(f_{I}\right)=\sum_{i \in I} r_{i}-\sum_{i \in I^{c}} r_{i}=\varepsilon_{I}(r) .
$$

Remark 3.5. Note that $\varepsilon_{I}(r) \neq 0$ for all $I$ because we assumed $r$ generic. This implies that 0 is a regular value of the moment map $\mu$. In fact $d_{x} \mu$ is identically 0 if and only if $x=f_{I}$ : for each tangent vector $v=\left(v_{1}, \ldots, v_{n}\right) \in$ $T_{x} \mathcal{S}, d_{x} \mu(v)=\sum_{j} \zeta_{j}$, where $\zeta_{j}$ is the third component of $v_{j}$. So $d_{x} \mu \equiv 0 \Longleftrightarrow$ $\zeta_{j}=0 \forall j \Longleftrightarrow x=f_{I}$ for some $I$.

From the Atiyah and Guillemin-Sternberg convexity Theorem, the image $\mu\left(\mathcal{S}_{r}\right)$ is the convex hull of the points $\mu\left(f_{I}\right)$, i.e.,

$$
\mu\left(\mathcal{S}_{r}\right)=\left[-\sum_{i=1}^{n} r_{i}, \sum_{i=1}^{n} r_{i}\right] .
$$

The idea is now to apply Theorem 3.1 to calculate the volume of $\mathcal{S}_{r} / / S^{1}$. Choose $p_{0}=0$ and $p_{1}>\sum_{i=1}^{n} r_{i}$, so that $\mu^{-1}\left(p_{1}\right)$ is empty, this implies that the integral over $\mathcal{S}_{r} / / S^{1}\left(p_{1}\right)$ is zero and

$$
\int_{\mathcal{S}_{r} / / S^{1}} k(\tilde{a})=\sum \int_{X^{H} / / T(q)} k_{q}\left(\lambda_{H}\left(\tilde{a}_{\left.\right|_{X} H}\right)\right)
$$

where the sum is made over the walls $\mu\left(X^{H_{i}}\right)$ that the path $Z=\left[0, p_{1}\right] \subset \mathbb{R}$ crosses at $q_{i}$.

Moreover, note that the walls in $\mu\left(\mathcal{S}_{r}\right)$ are just the points $\mu\left(f_{I}\right)$, and that the path $Z$ crosses the walls $\mu\left(f_{I}\right)$ only for those $I$ such that $\varepsilon_{I}(r)>0$. Let $\mathcal{I}$ be the family of all these index sets $I$. Since that the quotient spaces $X^{H} / / T(q)$ are just points, we obtain

$$
\int_{\mathcal{S}_{r} / / S^{1}} k(\tilde{a})=\sum_{I \in \mathcal{I}} k_{I}\left(\lambda_{I}\left(\tilde{a}_{\left.\right|_{I}}\right)\right) .
$$

Now we will study the normal bundle $\nu f_{I}$ in order to work out the necessary details to use the equivariant description of $\lambda_{f_{I}}$ (see Proposition 3.2).

The $f_{I}$ 's are points thus for each $I$ the normal bundle $\nu f_{I}$ is the direct sum of copies of $T_{N_{j}} S_{r_{j}}^{2}$ and $T_{S_{j}} S_{r_{j}}^{2}$. Precisely

$$
\nu f_{I} \simeq \mathbb{C}_{(1)}^{|I|} \oplus \mathbb{C}_{(-1)}^{n-|I|}
$$

The equivariant Segre classes that appear in Proposition 3.2 formally lie in $H_{T / H}^{*}\left(f_{I}\right)$, where $H \simeq S^{1}$ is the subgroup of $T$ associated to the wall $\mu\left(f_{I}\right)$; in our case $T$ is $S^{1}$ itself, so $s^{w}\left(\nu f_{I}\right)$ lies in the de Rham cohomology ring $H^{*}\left(f_{I}\right)$. The bundle $\nu f_{I}$ has rank one, and the $j$ th Chern classes $c_{j}\left(\mathbb{C}_{( \pm 1)}\right)$ 
are zero for each $I$ and $j$ (because, for each $I, \nu f_{I}$ is a line bundle over a point). Then

$$
c^{w}\left(\nu f_{I}\right)=(-1)^{n-|I|}
$$

and

$$
s_{j}^{w}\left(\nu f_{I}\right) \begin{cases}(-1)^{n-|I|} & j=0, \\ 0 & \text { otherwise. }\end{cases}
$$

Moreover, the greatest common divisor $k\left(f_{I}\right)=1$ for each $I$ because the weights are all \pm 1 .

We have now all the ingredients to apply the equivariant formula in Proposition 3.2 and calculate $\lambda_{I}\left(\tilde{a}_{\left.\right|_{f_{I}}}\right)$, with $\tilde{a}=c_{1}^{S^{1}}(\mathcal{L})^{n-3} \smile c_{1}^{S^{1}}\left(\underline{\mathbb{C}}_{(1)}\right) \smile$ $c_{1}^{S^{1}}\left(\underline{\mathbb{C}}_{(-1)}\right)$.

From the construction of the line bundle $\mathcal{L}$ we made above, it follows that $\mathcal{L}_{\left.\right|_{f_{I}}}=\mathbb{C}_{\left(\varepsilon_{I}(r)\right)}$ where again $I$ is the index set that "detects" the north poles. Thus

$$
c_{1}^{S^{1}}(\mathcal{L})_{f_{I}}=\left(\varepsilon_{I}(r)\right) u,
$$

where $u$ is the positive generator of the equivariant cohomology of a point $H_{S^{1}}^{*}\left(f_{I}\right)$. Similarly,

$$
c_{1}^{S^{1}}\left(\underline{\mathbb{C}}_{(1)}\right)_{\left.\right|_{f_{I}}}=u, \quad c_{1}^{S^{1}}\left(\underline{\mathbb{C}}_{(-1)}\right)_{\left.\right|_{f_{I}}}=-u .
$$

So

$$
\tilde{a}_{\left.\right|_{I}}=-\left(\varepsilon_{I}(r)\right)^{n-3} u^{n-1}
$$

and

$$
\lambda_{I}\left(\tilde{a}_{\left.\right|_{f_{I}}}\right)=-(-1)^{n-|I|}\left(\varepsilon_{I}(r)\right)^{n-3} u^{n-1} .
$$

To finish the proof we should now apply the Kirwan map $k_{q}: H_{T / H}^{*}\left(X^{H}\right) \rightarrow$ $H^{*}\left(X^{H} / / T(q)\right)$ as in Theorem 3.1. Since in our case $T$ is $S^{1}$ itself and the fixed points sets $X^{H}$ are the $f_{I}$ 's, the map $k_{q}: H^{*}\left(f_{I}\right) \rightarrow H^{*}\left(f_{I}\right)$ is the identity. Thus, summing on all the admissible $I$, the result follows.

3.3. Examples. Let $\Delta^{0}$ be the chamber in $\Xi \in \mathbb{R}^{5}$ determined by its collection of short sets

$$
\begin{aligned}
\mathcal{S}\left(\Delta^{0}\right)= & \{\{i\} \mid i=1, \ldots, 5\} \cup\{\{j, k\} \mid j, k=1,2,4,5\} \\
& \cup\{\{i, j, k\} \mid i, j, k=1,2,4,5\} .
\end{aligned}
$$

Consequently, the collection of $r^{0}$-long sets is

$$
\mathcal{I}\left(r^{0}\right)=\{\{3, j\},\{3, j, k\}: j, k=1,2,4,5\} \cup\{I \subseteq\{1, \ldots, 5\}:|I|=4,5\} .
$$


Note that the chamber $\Delta^{0}$ is not empty, for example, $\frac{1}{7}(1,1,3,1,1) \in \Delta^{0}$. Then, by Theorem 3.4, it follows (by plain computation) that the volume of the associated symplectic quotient $M\left(r^{0}\right)$ is

$$
\operatorname{vol} M\left(r^{0}\right)=2 \pi^{2}\left(r_{1}^{0}+r_{2}^{0}-r_{3}^{0}+r_{4}^{0}+r_{5}^{0}\right)^{2} .
$$

Because the perimeter $\sum_{i=1}^{n} r_{i}=1$ is fixed on $\Xi$, one also obtains $\operatorname{vol} M\left(r^{0}\right)=2 \pi^{2}\left(1-2 r_{3}^{0}\right)^{2}$.

Consider now the adjacent chamber $\Delta^{1}$ characterized by

$$
\begin{aligned}
\mathcal{S}\left(\Delta^{1}\right)= & \{\{i\} \mid i=1, \ldots, 5\} \cup\{\{j, k\} \mid j, k=1,2,4,5\} \cup\{1,3\} \\
& \cup\{\{1, j, k\} \mid j, k=2,4,5\} .
\end{aligned}
$$

Also the chamber $\Delta^{1}$ is not empty, as for example the lengths vector $\frac{2}{11}\left(\frac{1}{2}, 1,2,1,1\right)$ is in $\Delta^{1}$. The closures of $\Delta^{0}$ and $\Delta^{1}$ intersect in the wall of equation $\varepsilon_{\{1,3\}}(r)=\varepsilon_{\{2,4,5\}}(r)=0$. This means that $\{1,3\}$ is $r^{0}$-long and $r^{1}$-short, while its complement $\{2,4,5\}$ is $r^{1}$-long and $r^{0}$-short. This is the only difference between in the collections of $r^{0}$-long sets and $r^{1}$-long sets. Hence, applying Theorem 3.4, we get

$$
\operatorname{vol} M\left(r^{1}\right)=4 \pi^{2} r_{1}^{1}\left(r_{2}^{1}-r_{3}^{1}+r_{4}^{1}+r_{5}^{1}\right) \text {. }
$$

Again, using the fixed perimeter condition, one obtains $\operatorname{vol} M\left(r^{1}\right)=$ $4 \pi^{2} r_{1}^{1}\left(1-r_{1}^{1}-2 r_{3}^{1}\right)$.

\section{Crossing the walls}

In this section, we explicitly describe how the diffeotype of the manifold $M(r)$ changes as $r$ crosses a wall in $\Xi=\mu_{U(1)^{n}}(G r(2, n))$.

The chambers $\Delta^{i}$ of regular values in the convex polytope $\Xi$ are convex polytopes themselves. They are separated by walls, i.e., by the images $\mu_{U(1)^{n}}\left(G r(2, n)^{S^{1}}\right)$ of the sets of points fixed by the circle subgroups of $U(1)^{n}$. For $r^{0}$ and $r^{1}$ in different chambers $\Delta^{0}$ and $\Delta^{1}$, the symplectic reductions $M\left(r^{0}\right)$ and $M\left(r^{1}\right)$ are related by a birational map that can be described in terms of blowing up and down submanifolds. This follows from a general construction due independently to Brion-Procesi $[\mathbf{B P}]$ and to GuilleminSternberg [GS89]. In this section, we show that these submanifolds are resolutions of the singularity corresponding to the degenerate polygon in the singular quotient $M\left(r^{c}\right)$ (where $r^{c}$ is the wall-crossing point) and characterize them in terms of polygon spaces of lower dimension.

Through all the paper we assume a single wall-crossing, meaning that the wall-crossing point is not on a intersection of walls, but lies on one and only one wall. This also implies that the quotient $M\left(r^{c}\right)$ has only one critical point. The assumption is not restrictive since any non-single wall-crossing can be decomposed in a finite number of subsequent single wall-crossing; cf. [GS89]. 
In [GS89] Guillemin and Sternberg give a thorough analysis of wallcrossing problems relative to (quasi-free) $S^{1}$-actions. They also point out that their construction can be made $H$-equivariant, when $H$ is any compact group commuting with the $S^{1}$-action. This is our case: in fact we will first perform the symplectic reduction by a complement $H$ of the $S^{1}$ associated to the wall and then apply the analysis as in $\left[\mathbf{G S 8 9}\right.$ ] to the remaining $S^{1}$ action. Still there is a small subtlety here, since the action of $U(1)^{n}$ is not effective.

In this section, we prove the following Theorem 4.1. Before stating that, let us introduce some notation: consider $r^{0}$ and $r^{1}$ regular values of $\mu_{U(1)^{n}}$ lying in different chambers, $\Delta^{0}$ and $\Delta^{1}$ respectively, separated by the wall of equation

$$
\varepsilon_{I_{p}}(r)=0
$$

Assume also that the lengths vectors $r^{0} \in \Delta^{0}$ and $r^{1} \in \Delta^{1}$ satisfy

$$
\varepsilon_{I_{p}}\left(r^{0}\right)>0 \text { and } \varepsilon_{I_{p}}\left(r^{1}\right)<0
$$

and call $W_{I_{p}}$ the wall of Equation (4.1) together with the wall-crossing direction from $\Delta^{0}$ to $\Delta^{1}$. Moreover, for any index set $I \subset\{1, \ldots n\}$, let $M_{I}(r)$ be the (eventually empty) submanifold of $M(r)$ of those polygons such that the edges $e_{i}$, for $i \in I$, are positive proportional to each other. Precisely

$$
M_{I}(r):=\widetilde{M}_{I}(r) / S O(3),
$$

where

$$
\widetilde{M}_{I}(r):=\left\{\left(e_{1}, \ldots, e_{n}\right) \in \mathcal{S}_{r} \mid \sum_{j=1}^{n} e_{j}=0, e_{i}=\lambda_{k} e_{k}, \forall i, k \in I, \lambda_{k} \in \mathbb{R}_{+}\right\}
$$

Theorem 4.1. Let the lengths vector $r$ cross a wall $W_{I_{p}}$ in $\Xi$ as above. Then the diffeotype of the moduli space of polygons $M(r)$ changes by blowing up $M_{I_{p}^{c}}\left(r^{0}\right) \simeq \mathbb{C P}^{p-2}$ and blowing down the projectivized normal bundle of $M_{I_{p}}\left(r^{1}\right) \simeq \mathbb{C P}^{q-2}$.

The polygon spaces $M_{I_{p}}\left(r^{1}\right)$ and $M_{I_{q}}\left(r^{0}\right)$ are resolutions of the singularity corresponding to the degenerate polygon $\left[P^{c}\right]$ in $M\left(r^{c}\right)$, and both are dominated by the blow-up $\widetilde{M}$ of $M\left(r^{c}\right)$ at the singular point, with exceptional divisor $\mathbb{C P}^{p-2} \times \mathbb{C P}^{q-2}$.

Proof. Let $H^{\prime}:=\left\{\operatorname{diag}\left(e^{i \beta_{1}}, \ldots, e^{i \beta_{n-1}}, 1\right) \mid e^{i \beta_{j}} \in S^{1} \quad \forall j=1, \ldots, n-1\right\} \subset$ $U(1)^{n}$ be a complement of the diagonal circle $\left\{\operatorname{diag}\left(e^{i \theta}, \ldots, e^{i \theta}\right)\right\} \subset U(1)^{n}$. The group $H^{\prime}$ acts effectively on $\operatorname{Gr}(2, n)$ by restriction of the $U(1)^{n}$-action 
with associated moment map

$$
\begin{aligned}
\mu_{H^{\prime}}: G r(2, n) & \rightarrow \mathbb{R}^{n-1}, \\
(a, b) & \mapsto \frac{1}{2}\left(\left|a_{1}\right|^{2}+\left|b_{1}\right|^{2}, \ldots,\left|a_{n-1}\right|^{2}+\left|b_{n-1}\right|^{2}\right) .
\end{aligned}
$$

The moment polytope $\mu_{H^{\prime}}(G r(2, n))$ is the image of $\Xi$ via the projection $\operatorname{map} \mathbb{R}^{n} \rightarrow \mathbb{R}^{n-1}$ that drops the last coordinate. Since the action of $U(1)^{n}$ is not effective (the diagonal circle fixes every point), one can easily see that both the quotients

$$
\mu_{U(1)^{n}}^{-1}\left(r_{1}, \ldots, r_{n}\right) / U(1)^{n} \quad \text { and } \quad \mu_{H^{\prime}}^{-1}\left(r_{1}, \ldots, r_{n-1}\right) / H^{\prime}
$$

are diffeomorphic, and each is the moduli space of polygons $M(r)$. Note in particular that $r_{n}$ is uniquely determined by $r_{1}, \ldots, r_{n-1}$. In other words, if $\left(r_{1}, \ldots, r_{n}\right)$ are coordinates in $\Xi$, then the coordinates $r_{1}, \ldots, r_{n-1}$ on the projected polytope $\mu_{H^{\prime}}(G r(2, n))$ satisfy

$$
r_{n}=1-\sum_{i=1}^{n-1} r_{i} .
$$

It follows that the wall $\varepsilon_{I_{p}}(r)=0$ is mapped in $\mu_{H^{\prime}}(\operatorname{Gr}(2, n))$ onto the wall of equation

$$
\sum_{i \in I_{p}} r_{i}=\frac{1}{2}
$$

In particular, external walls satisfy $2 \sum_{i \in I_{p}} r_{i}=1$ for $I_{p}$ of cardinality 1 or $n-1$. Note that whenever (4.4) holds, then the condition $\sum_{1}^{n} r_{i}=1$ implies $2 \sum_{i \in I_{p}^{c}} r_{i}=1$, where $I_{p}^{c}$ is the complement of $I_{p}$ in $\{1, \ldots, n\}$. Therefore, there exists at least one index $i \in I_{p}$ and at least one index $j \in I_{p}^{c}$ such that $r_{i} \neq 0$ and $r_{j} \neq 0$. Since $M(r)$ is symplectomorphic to $M(\sigma(r))$ for any permutation $\sigma$ of the $n$ edges, it is not restrictive to assume $I_{p}=\{1, \ldots p\}$ and $r_{1} \neq 0$ as well as $r_{n} \neq 0$.

The orientation of the circle associated to $W_{I_{p}}$ is determined accordingly with the wall-crossing direction. This means that among the directions

$$
v^{ \pm}= \pm(\underbrace{-1, \ldots,-1}_{p}, 0, \ldots, 0)
$$

normal to the wall (4.4), we wish to choose the one that has positive inner product with the vector $\left(r^{1}-r^{0}\right) \in \mathbb{R}^{n-1}$. This is the case for $v^{-}$(it follows from assumption (4.2)), and therefore the circle associated to $W_{I_{p}}$ is

$$
S^{1}:=\left\{\operatorname{diag}(\underbrace{e^{-i \theta}, \ldots, e^{-i \theta}}_{p}, 1, \ldots, 1)\right\} \subset H^{\prime} \subset U(1)^{n} .
$$


Let $r^{c}$ be the wall-crossing point and let

$$
H:=\left\{\operatorname{diag}\left(1, e^{i \theta_{2}}, \ldots, e^{i \theta_{n-1}}, 1\right) \mid e^{i \theta_{j}} \in S^{1} \forall j=2, \ldots, n-1\right\}
$$

be a complement of $S^{1}$ in $H^{\prime}$. The group $H$ acts on $\operatorname{Gr}(2, n)$ in a Hamiltonian fashion with associated moment map

$$
\mu_{H}(a, b)=\frac{1}{2}\left(\left|a_{2}\right|^{2}+\left|b_{2}\right|^{2}, \ldots,\left|a_{n-1}\right|^{2}+\left|b_{n-1}\right|^{2}\right) .
$$

We now analyze the $S^{1}$-action on $\mu_{H}^{-1}\left(r_{2}^{c}, \ldots, r_{n-1}^{c}\right) / H$. In particular, if $\mu_{S^{1}}$ is the moment map for the residual $S^{1}$-action on $\mu_{H}^{-1}\left(r_{2}^{c}, \ldots, r_{n-1}^{c}\right) / H$, we will describe the singular reduced space $\mu_{S^{1}}^{-1}(0) / S^{1}$ as in $[\mathbf{G u}$ ], obtaining also the two resolutions of the singularity in Theorem 4.1.

To this aim, note that the fixed points set of the $S^{1}$-action consists only of the point $\left[P^{c}\right]$ :

$$
\left(\mu_{H}^{-1}\left(r_{2}^{c}, \ldots, r_{n-1}^{c}\right) / H\right)^{S^{1}}=\left[P^{c}\right]:=\left(\begin{array}{cc}
\sqrt{1-2 \sum_{2}^{p} r_{i}^{c}} & 0 \\
\sqrt{2 r_{2}^{c}} & 0 \\
\vdots & \vdots \\
\sqrt{2 r_{p}^{c}} & 0 \\
0 & \sqrt{2 r_{p+1}^{c}} \\
\vdots & \vdots \\
0 & \sqrt{2 r_{n-1}^{c}} \\
0 & \sqrt{1-2 \sum_{p+1}^{n-1} r_{i}^{c}}
\end{array}\right) .
$$

To prove (4.5) note that an element $(a, b) \in \mu_{H}^{-1}\left(r_{2}^{c}, \ldots, r_{n-1}^{c}\right) / H$ is fixed by the $S^{1}$-action if and only if

$$
\left\{\begin{array}{l}
a_{i}=0, \quad \forall i=p+1, \ldots, n, \\
b_{j}=0 \quad \forall j=1, \ldots, p .
\end{array}\right.
$$

It then follows from the moment map conditions that $\left|a_{i}\right|=\sqrt{2 r_{i}^{c}}$ for all $i=$ $2, \ldots, n$ and $\left|b_{j}\right|=\sqrt{2 r_{j}^{c}}$ for all $j=p+1, \ldots, n$. Recalling that $\sum_{1}^{n}\left|a_{i}\right|^{2}=1$ and $\sum_{1}^{n}\left|b_{i}\right|^{2}=1$ since $(a, b) \in S t_{2, n}$, we get that

$$
a_{1}=\sqrt{1-2 \sum_{2}^{p} r_{i}^{c}} e^{i \phi_{1}} \text { and } b_{n}=\sqrt{1-2 \sum_{p+1}^{n-1} r_{i}^{c}} e^{i \phi_{n}}
$$

for some $\phi_{1}, \phi_{n} \in\left[0,2 \pi\left[\right.\right.$. Modulo the $S U(2)$-action, we can then take $a_{1}$ and $b_{n}$ to be real. Now modulo the $H$ action, we can take $e^{-i \phi_{1}} a_{i}, i=2, \ldots, p$, and $e^{i \phi_{n}} b_{j}, j=p+1, \ldots, n-1$, to be real as well, and (4.5) follows.

In a neighborhood $\mathcal{U} \subset \mu_{H}^{-1}\left(r_{2}^{c}, \ldots, r_{n-1}^{c}\right) / H$ of the fixed point $\left[P^{c}\right]$ we give a local system of coordinates $\left(w_{2}, \ldots, w_{p}, z_{p+1}, \ldots, z_{n-1}\right) \in \mathbb{C}^{n-2}$, centered 
at $\left[P^{c}\right]$, such that

$$
P=\left(\begin{array}{cc}
l_{1} & 0 \\
l_{2} & w_{2} \\
\vdots & \vdots \\
l_{p} & w_{p} \\
z_{p+1} & m_{p+1} \\
\vdots & \vdots \\
z_{n-1} & m_{n-1} \\
0 & m_{n}
\end{array}\right) \quad \forall P \in \mathcal{U}
$$

with $l_{j}$ and $m_{k}$ real functions of $(w, z)$ and of the wall-crossing value $r^{c}$. These local coordinates can be determined as follows. Given $(a, b) \in \mathcal{U}$ consider a non-zero minor, for example the one formed by the first and the last row (this clearly does not vanish for $\mathcal{U}$ neighborhood of $\left[P^{c}\right]$ small enough). Then using the $U(2)$ action we can rewrite $(a, b)$ as

$$
\left(\begin{array}{cc}
\tilde{l}_{1} & 0 \\
\tilde{z}_{2} & \tilde{w}_{2} \\
\vdots & \vdots \\
\tilde{z}_{n-1} & \tilde{w}_{n-1} \\
0 & \tilde{m}_{n}
\end{array}\right)
$$

where $\tilde{l}_{1}=\left|a_{1}\right|^{2}+\left|b_{1}\right|^{2}, \tilde{m}_{n}=\left|a_{n}\right|^{2}+\left|b_{n}\right|^{2}$ and

$$
\tilde{z}_{i}=\frac{a_{i} b_{n}-a_{n} b_{i}}{a_{1} b_{n}-a_{n} b_{1}}, \quad \tilde{w}_{i}=\frac{a_{1} b_{i}-a_{i} b_{1}}{a_{1} b_{n}-a_{n} b_{1}} .
$$

Writing $\tilde{z}_{j}=\left|\tilde{z}_{j}\right| e^{i \tilde{\theta}_{j}}$ for $j=2, \ldots, p$ and $\tilde{w}_{k}=\left|\tilde{w}_{k}\right| e^{i \tilde{\theta}_{k}}$ for $k=p+1, \ldots, n-$ 1 , one can see that, modulo the $H$-action, (4.7) becomes (4.6), where

$$
\begin{aligned}
& w_{j}=e^{-i \tilde{\theta}_{j}} \tilde{w}_{j}, \quad l_{j}=\left|\tilde{z}_{j}\right|=\sqrt{2 r_{j}^{c}-\left|w_{j}\right|^{2}}, \quad \forall j=2, \ldots, p, \\
& z_{k}=e^{-i \tilde{\theta}_{k}} \tilde{z}_{k}, \quad m_{k}=\left|\tilde{w}_{k}\right|=\sqrt{2 r_{k}^{c}-\left|z_{k}\right|^{2}} \quad \forall k=p+1, \ldots, n-1
\end{aligned}
$$

and consequently

$$
\begin{aligned}
l_{1} & =\left(1-2 \sum_{j=2}^{p} r_{j}^{c}+\sum_{j=2}^{p}\left|w_{j}\right|^{2}-\sum_{k=p+1}^{n-1}\left|z_{k}\right|^{2}\right)^{1 / 2} \\
m_{n} & =\left(1-2 \sum_{k=p+1}^{n-1} r_{k}^{c}-\sum_{j=2}^{p}\left|w_{j}\right|^{2}+\sum_{k=p+1}^{n-1}\left|z_{k}\right|^{2}\right)^{1 / 2} .
\end{aligned}
$$


In such a neighborhood $\mathcal{U}$ of $\left[P^{c}\right]$ the action of $S^{1}$ is then

$$
\begin{aligned}
& \operatorname{diag}\left(e^{-i \theta}, \ldots, e^{-i \theta}, 1, \ldots, 1\right) \cdot\left(w_{2}, \ldots, w_{p}, z_{p+1}, \ldots, z_{n-1}\right) \\
& \quad=\left(e^{-i \theta} w_{2}, \ldots, e^{-i \theta} w_{p}, e^{i \theta} z_{p+1}, \ldots, e^{i \theta} z_{n-1}\right)
\end{aligned}
$$

with associated moment map

$$
\mu_{S^{1}}\left(w_{2}, \ldots, w_{p}, z_{p+1}, \ldots, z_{n-1}\right)=\frac{1}{2}\left(-\sum_{j=2}^{p}\left|w_{j}\right|^{2}+\sum_{k=p+1}^{n-1}\left|z_{k}\right|^{2}\right) .
$$

The critical level set

$$
\sum_{j=2}^{p}\left|w_{j}\right|^{2}=\sum_{k=p+1}^{n-1}\left|z_{k}\right|^{2}
$$

is a conic subset of $\mathbb{C}^{n-2}$. Precisely, it is the cone over the product of the two spheres $S^{2 p-3}=\left\{\left.w_{j}\left|\sum_{j=2}^{p}\right| w_{j}\right|^{2}=1\right\}$ and $S^{2 q-3}=\left\{\left.z_{k}\left|\sum_{k=p+1}^{n-1}\right| z_{k}\right|^{2}=\right.$ $1\}$, where $q:=n-p$. The action of $S^{1}$ on this product is free so the orbit space $W=\left(S^{2 p-3} \times S^{2 q-3}\right) / S^{1}$ is a compact manifold and the quotient $\mu_{S^{1}}^{-1}(0) / S^{1}$ in the neighborhood $\mathcal{U}$ of $\left[P^{c}\right]$ looks like a cone $C_{W}$ over $W$ with vertex at $\{0\}=\left[P^{c}\right]$.

From the action $\left(w_{2}, \ldots, w_{p}\right) \mapsto\left(e^{-i \theta} w_{2}, \ldots, e^{-i \theta} w_{p}\right)$ of $S^{1}$ on $S^{2 p-3}$ one gets the Hopf fibration

$$
\pi: S^{2 p-3} \rightarrow \mathbb{C P}^{p-2} .
$$

Since $S^{1}$ acts also on $S^{2 q-3}$, one can consider the associated bundle

$$
\left(S^{2 p-3} \times S^{2 q-3}\right) / S^{1} \rightarrow \mathbb{C P}^{p-2}
$$

obtaining a description of $W$ as a fiber bundle over $\mathbb{C} \mathbb{P}^{p-2}$. Reversing the roles of $p$ and $q$ we get $W$ as a fiber bundle over $\mathbb{C P}^{q-2}$. Associated with these two description of $W$ we obtain the desingularizations of $M\left(r^{c}\right)$ as in Theorem 4.1. In fact, since the action of $S^{1}$ on $S^{2 q-3}$ extends to a linear action on $\mathbb{C}^{q-1}$ one can form the associated vector bundle

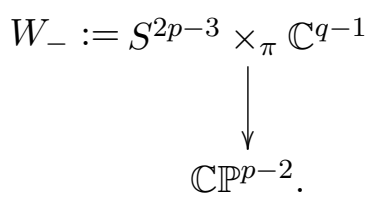

On this bundle there is a blowing down map $\beta_{-}: W_{-} \rightarrow C_{W}$,

$$
\begin{aligned}
\beta_{-} & \left(w_{2}, \ldots, w_{p}, z_{p+1}, \ldots, z_{n-1}\right) \\
& =\left(\sqrt{\sum_{i=p+1}^{n-1}\left|z_{i}\right|^{2}} w_{2}, \ldots, \sqrt{\sum_{i=p+1}^{n-1}\left|z_{i}\right|^{2}} w_{p}, z_{p+1}, \ldots, z_{n-1}\right)
\end{aligned}
$$


and an embedding of $\mathbb{C P}^{p-2}$ as the zero section of the bundle (4.9):

$$
\begin{aligned}
\iota: \mathbb{C P}^{p-2} & \rightarrow W_{-} \\
{\left[w_{2}, \ldots, w_{p}\right] } & \mapsto\left(w_{2}, \ldots, w_{p}, 0 \ldots, 0\right) .
\end{aligned}
$$

Moreover, the image of $\mathbb{C P}^{p-2}$ in $W_{-}$gets blown down to $\{0\} \in C_{W}$, and $\beta_{-}$maps $W_{-} \backslash \mathbb{C P}^{p-2}$ diffeomorphically onto $C_{W} \backslash\{0\}$.

On the other hand, reversing again the roles of $p$ and $q$ one obtains a desingularization $W_{+}$of $M\left(r^{c}\right)$, where $W_{+}$is now the bundle

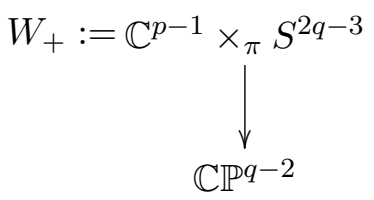

with associated blowing down map $\beta_{+}: W_{+} \rightarrow C_{W}$ and embedding $\iota$ : $\mathbb{C P}^{q-2} \rightarrow W_{+}$as the zero section.

Via these two desingularizations we obtain our description of the birational map from $M\left(r^{0}\right)$ to $M\left(r^{1}\right)$ with $r^{0}$ and $r^{1}$ as in Theorem 4.1.

In fact, for $-\sum_{j=2}^{p}\left|w_{j}\right|^{2}+\sum_{k=p+1}^{n-1}\left|z_{k}\right|^{2}=-\varepsilon$ the orbit space $\mu_{S^{1}}^{-1}(-\varepsilon) / S^{1}$ is identical topologically with $W_{-}$. Note that $r_{1}$ is then uniquely determined

$$
r_{1}=l_{1}^{2}=1-\sum_{j=2}^{p} r_{j}^{c}+\varepsilon
$$

and consequently

$$
r_{n}=1-\sum_{k=p+1}^{n-1} r_{k}^{c}-\varepsilon .
$$

This means that $r^{0}:=\left(r_{1}, r_{2}^{c}, \ldots, r_{n-1}^{c}, r_{n}\right)$, with $r_{1}$ and $r_{n}$ as above, satisfies $\varepsilon_{I_{p}}\left(r^{0}\right)>0$. Using the right-hand side of diagram (2.3) we can give a geometric characterization of the $\mathbb{C P}^{p-2}$ that is blown down by the map $\beta_{-}$, describing it as a lower-dimensional polygon space.

As seen above, $\mathbb{C P}^{p-2}$ is embedded in $W_{-}$as the zero section with respect to the local coordinates $(w, z)$, i.e.,

$$
\mathbb{C P}^{p-2}=\left\{\left(w_{1}, \ldots, w_{p}, z_{p+1}, \ldots, z_{n-1}\right) \mid z_{k}=0 \forall k=p+1, \ldots, n-1\right\} .
$$

From (4.8) it follows

$$
z_{k}=0 \Longleftrightarrow a_{k} b_{n}-a_{n} b_{k}=0
$$

We now want to describe $\mathbb{C P}^{p-2}$ performing the reductions as the right-hand side of the diagram (2.3). To this aim, start from $(a, b) \in p^{-1}\left(\mu_{U(1)^{n}}^{-1}(r)\right) \subset$ $S t_{2, n}$ satisfying (4.12) for every $k=p+1, \ldots, n-1$ and consider it as an 
element in $\prod_{j} S_{\sqrt{2 r_{j}}}^{3}$ via the inclusion map $\imath$ as in (2.2). Recall that the Hopf map $H^{n}$ maps $(a, b)$ to $\left(e_{1}, \ldots, e_{n}\right) \in \prod_{j} S_{r_{j}}^{2}$ where $e_{i}=1 / 2\left(\left|a_{i}\right|^{2}-\right.$ $\left.\left|b_{i}\right|^{2}, 2 \operatorname{Re}\left(\bar{a}_{i} b_{i}\right), 2 \operatorname{Im}\left(\bar{a}_{i} b_{i}\right)\right)$.

Condition (4.12) then implies that for every $k=p+1, \ldots, n-1$ the vectors $e_{k}$ are positive multiples of each other, i.e.,

$$
\forall k=p+1, \ldots, n-1, \exists \lambda_{k} \in \mathbb{R}_{+} \text {s.t. } e_{k}=\lambda_{k} e_{n} .
$$

In fact, if $a_{n}=0$ then $a_{k}=0$ (note that $b_{n}$ cannot vanish simultaneously with $a_{n}$ since we assumed $\left.r_{n} \neq 0\right)$ and clearly

$$
e_{k}=\left(-\frac{\left|b_{k}\right|^{2}}{2}, 0,0\right)=\frac{\left|b_{k}\right|^{2}}{2\left|b_{n}\right|^{2}}\left(-\left|b_{n}\right|^{2}, 0,0\right)=\frac{\left|b_{k}\right|^{2}}{2\left|b_{n}\right|^{2}} e_{n} .
$$

Similarly, if $b_{n}=0$ then

$$
e_{k}=\left(\frac{\left|a_{k}\right|^{2}}{2}, 0,0\right)=\frac{\left|a_{k}\right|^{2}}{2\left|a_{n}\right|^{2}} e_{n} .
$$

If both $a_{n}$ and $b_{n}$ are non-zero, from (4.12) we obtain $b_{k} \bar{a}_{k}=\frac{b_{n}}{a_{n}}\left|a_{k}\right|^{2}$. This implies

$$
\left|b_{k}\right|^{2}=\frac{\bar{b}_{n}}{\bar{a}_{n}} \bar{a}_{k} b_{k}=\frac{\left|\bar{b}_{n}\right|^{2}}{\left|\bar{a}_{n}\right|^{2}}\left|a_{k}\right|^{2}
$$

It then follows that

$$
e_{k}=\left|a_{k}\right|^{2}\left(\frac{1}{2}\left(1-\frac{\left|\bar{b}_{n}\right|^{2}}{\left|\bar{a}_{n}\right|^{2}}\right), \operatorname{Re} \frac{b_{n}}{a_{n}}, \operatorname{Im} \frac{b_{n}}{a_{n}}\right)=\frac{\left|a_{k}\right|^{2}}{2\left|\bar{a}_{n}\right|^{2}} e_{n}
$$

proving (4.13).

Therefore,

$$
\begin{aligned}
& H^{n}\left(\imath \left\{(a, b) \in p^{-1}\left(\mu_{U(1)^{n}}^{-1}(r)\right) \mid a_{k} b_{n}-a_{n} b_{k}=0\right.\right. \\
& \forall k=p+1, \ldots, n-1\})=\widetilde{M}_{I_{p}^{c}}(r)
\end{aligned}
$$

and the projective space (4.11) is then the quotient $M_{I_{p}^{c}}(r):=\widetilde{M}_{I_{p}^{c}}(r) / S O(3)$. In words, $M_{I_{p}^{c}}(r)$ is the submanifold of $M(r)$ of those $n$-gons such that the last $n-p$ edges are positive multiple one of the other. Note that for $r \in \Delta^{0}$ this is a non-empty submanifold (in fact it is a $\mathbb{C P}^{p-2}$ ). In particular, $M_{I_{p}^{c}}(r)$ is naturally diffeomorphic to the $(p-2)$-dimensional polygon space $M\left(r_{1}, \ldots, r_{p}, \sum_{k=p+1}^{n} r_{k}\right)$.

On the other hand, for

$$
-\sum_{j=2}^{p}\left|w_{j}\right|^{2}+\sum_{k=p+1}^{n-1}\left|z_{k}\right|^{2}=\varepsilon
$$


the orbit space $\mu_{S^{1}}^{-1}(\varepsilon) / S^{1}$ is identical topologically with $W_{+}$. Again $r_{1}$ and $r_{n}$ are uniquely determined:

$$
r_{1}=1-\sum_{j=2}^{p} r_{j}^{c}-\varepsilon \quad \text { and } \quad r_{n}=1-\sum_{k=p+1}^{n-1} r_{k}^{c}+\varepsilon .
$$

The resulting lengths vector $r^{1}=\left(r_{1}, r_{2}^{c}, \ldots, r_{n-1}^{c}, r_{n}\right)$ satisfies $-\varepsilon_{I_{p}}\left(r^{1}\right)=$ $\varepsilon_{I_{p}^{c}}\left(r^{1}\right)>0$. The zero section of $W_{+}$is now the projective space $\mathbb{C P}^{q-2}$ corresponding to the vanishing of the coordinates $w_{j}$ for $j=2, \ldots, p$. It follows then from (4.8) that $w_{j}$ vanishes if and only if $a_{1} b_{j}-a_{j} b_{1}=0$. Arguments similar to the ones above allow us to identify $\mathbb{C P}^{q-2}$ with the submanifold $M_{I_{p}}(r) \subset M(r)$. Again, for $r \in \Delta^{1}$ the submanifold $M_{I_{p}}(r)$ is non-empty and is diffeomorphic to the $(q-2)$-dimensional polygon space $M\left(\sum_{j=1}^{p} r_{j}, r_{2}, \ldots, r_{n}\right)$.

Note that on the wall-crossing point $r^{c}, \varepsilon_{I_{p}}\left(r^{c}\right)=0$ and $M_{I_{p}}\left(r^{c}\right)=M_{I_{p}^{c}}\left(r^{c}\right)$ is the singular point $\left[P^{c}\right]$ in $M\left(r^{c}\right)$. Moreover, note that as $r^{0} \rightarrow r^{c}$, we have $\varepsilon_{I_{p}}\left(r^{0}\right) \rightarrow 0$ and $M_{I_{p}^{c}}\left(r^{0}\right) \subset M\left(r^{0}\right)$ collapses to $\left[P^{c}\right] \in M\left(r^{c}\right)$. Similarly, as $r^{1} \rightarrow r^{c}$, we have $\varepsilon_{I_{p}}\left(r^{1}\right) \rightarrow 0$ and $M_{I_{p}}\left(r^{1}\right) \subset M\left(r^{1}\right)$ collapses to $\left[P^{c}\right] \in$ $M\left(r^{c}\right)$. Roughly speaking, as $r^{0} \rightarrow r^{c}$, the "width" $\varepsilon_{I_{p}}\left(r^{0}\right)$ of polygons in $M_{I_{p}^{c}}\left(r^{0}\right) \subset M\left(r^{0}\right)$ goes to zero, and the $(p-2)$-dimensional submanifold $M_{I_{p}^{c}}(r)$ collapses to a point when $r$ reaches the wall $W_{I_{p}}$. Similarly, as $r$ leaves from the wall $W_{I_{p}}$ to the interior of $\Delta^{1}$, the degenerate polygon $\left[P^{c}\right]$ gets inflated of an $\varepsilon_{I_{p}^{c}}\left(r^{1}\right)$ amount, and $M_{I_{p}}\left(r^{1}\right)$ is the $(q-2)$-dimensional submanifold that is born as crossing the wall $W_{I_{p}}$.

The birational map between $M\left(r^{0}\right)$ and $M\left(r^{1}\right)$ is hence the composite of a blow-up followed by a blow-down, where the exceptional divisor is the product of the flip loci. The maps $\beta_{+}$and $\beta_{-}$blow down the flip loci $M_{I_{p}^{c}}(r)$ and $M_{I_{p}}(r)$ to the singular point $\left[P^{c}\right] \in M\left(r^{c}\right)$, as in Figure 2. Note that in Figure 2 there are no moment polytopes, just schematic representations of the (eventually singular) manifolds.

Note that the above wall-crossing analysis also holds for external walls. So in particular, for any $i=1, \ldots, n$, crossing the wall $W_{\{1, \ldots, n\} \backslash\{i\}}$ replaces the empty set with $M(r) \simeq M_{\{i\}}(r) \simeq \mathbb{C P}^{n-3}$. Therefore we have the following immediate consequence of Theorem 4.1

Proposition 4.2. Let $r$ be in an external chamber $\Delta$ of $\Xi$. Then

$$
M(r) \simeq \mathbb{C P}^{n-3} \text {. }
$$

In particular, Proposition 4.2 implies that for $n \geq 5$ the Weyl group acts transitively on the external chambers of $\Xi$. In fact, for $n \geq 5$, Farber, Hausmann and Schütz [FHS] have shown that, for $r$ 's in different chambers of $\Xi$, the polygon spaces $M(r)$ are not diffeomorphic unless their chambers are related by the Weyl group action. 


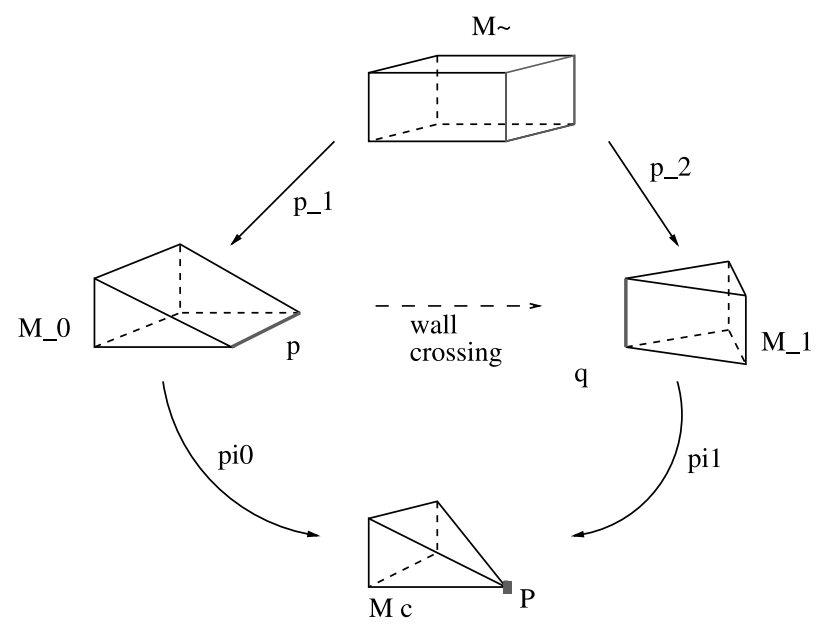

Figure 2. Crossing a wall.

4.1. Examples. Let $\Delta^{0}$ and $\Delta^{1}$ be the adjacent chambers in $\Xi \in \mathbb{R}^{5}$ as in Example 3.3. Note that $\{3\}$ is a maximal short set in $\mathcal{S}\left(\Delta^{0}\right)$ and therefore $\Delta^{0}$ is an external chamber. By Corollary $4.2, M\left(r^{0}\right) \simeq \mathbb{C P}^{2}$.

The closures of $\Delta^{0}$ and $\Delta^{1}$ intersect in the wall of equation

$$
\varepsilon_{\{1,3\}}(r)=\varepsilon_{\{2,4,5\}}(r)=0,
$$

see Example 3.3. In particular, if one considers the lengths vectors $r^{0}=$ $\frac{1}{7}(1,1,3,1,1) \in \Delta^{0}$ and $r^{1}=\frac{2}{11}\left(\frac{1}{2}, 1,2,1,1\right) \in \Delta^{1}$, then the segment $\left[r^{0}, r^{1}\right]$ hits the wall at $r^{c}=\frac{1}{6}\left(\frac{2}{3}, 1, \frac{7}{3}, 1,1\right)$. By Theorem 4.1, when crossing this wall the point $M_{\{2,4,5\}}\left(r^{0}\right) \simeq M\left(r_{2}^{0}+r_{4}^{0}+r_{5}^{0}, r_{1}^{0}, r_{3}^{0}\right)$ gets blown up. Therefore, for all $r^{1} \in \Delta^{1}, M\left(r^{1}\right)$ is diffeomorphic to $\mathbb{C P}^{2}$ blown up in one point with exceptional divisor $M_{\{1,3\}}\left(r^{1}\right) \simeq M\left(r_{1}^{1}+r_{3}^{1}, r_{2}^{1}, r_{4}^{1}, r_{5}^{1}\right) \simeq \mathbb{C P}^{1}$. The maps $\beta_{+}$and $\beta_{-}$blow down $M_{\{2,4,5\}}\left(r^{0}\right)$ and $M_{\{1,3\}}\left(r^{1}\right)$ to the critical point $\left[P^{c}\right]$ where the degenerate polygon has edges

$$
e_{1}^{c}=\left(\frac{1}{9}, 0,0\right), \quad e_{2}^{c}=\left(-\frac{1}{6}, 0,0\right)=e_{4}^{c}=e_{5}^{c}, \quad e_{3}^{c}=\left(\frac{7}{18}, 0,0\right) .
$$

\section{The cohomology ring of $M(r)$}

In this section, we study how the cohomology ring of $M(r)$ changes as $r$ crosses a wall in the moment polytope $\Xi$. We apply the DuistermaatHeckman Theorem together with the volume formula (Theorem 3.4) to describe explicitly the cohomology ring $H^{*}(M(r))$.

The study of the cohomology ring structure of a reduced space $M / / G$ has been since the 80 's one of the foremost topics in equivariant symplectic geometry. The problem is not closed though. In fact, (even in the well-behaved 
case of a compact connected Lie group $G$ acting on a compact manifold $M)$ to give an explicit description of the cohomology ring $H^{*}(M / / G, \mathbb{C})$ still some (non-trivial) work needs to be done. This was already pointed out by Guillemin and Sternberg in [GS95], who observed that in "nice" situations (essentially when the Chern class of the fibration $\mu^{-1}(\xi) \rightarrow M / / G$ generates the cohomology ring), then a good deal of information on $H^{*}(M / / G, \mathbb{C})$ can be deduced from the Duistermaat-Heckman Theorem, if the polynomial that describes the volume of the symplectic reduction is known. This is the point of view we take in our analysis.

5.1. The cohomology ring of reduced spaces. In this section, we summarize the main ideas and theorems in [GS95] using the notation of moduli spaces of polygons. These arguments are valid in more general settings, and have been applied in [GS95] to flag manifolds and toric manifolds associated with a simplicial fan. For proofs and more details we refer to [GS95]. Let $r$ and $r^{0}$ be regular values of $\mu_{U(1)^{n}}$ lying in the same chamber and denote by $\left(M(r), \omega_{r}\right)$ and $\left(M\left(r^{0}\right), \omega_{r^{0}}\right)$ the associated symplectic quotients. Using this notation we now state the Duistermaat-Heckman Theorem, which relates the cohomology classes $\left[\omega_{r}\right]$ and $\left[\omega_{r^{0}}\right]$ of the symplectic reduced forms $\omega_{r}$ and $\omega_{r^{0}}$.

Theorem 5.1 (J.J. Duistermaat, G.J. Heckman, [DH]). As differentiable manifolds $M(r)=M\left(r^{0}\right)$, and

$$
\left[\omega_{r}\right]=\left[\omega_{r^{0}}\right]+\sum_{i=1}^{n}\left(r_{i}-r_{i}^{0}\right) c_{i}
$$

where $c_{1}, \ldots, c_{n}$ are the first Chern classes of the $n$ line bundles associated to the fibration $\mu^{-1}(r) \rightarrow M(r)$.

By definition of symplectic volume, we have:

$$
\operatorname{vol} M(r)=\int_{M(r)} \exp \left(\left[\omega_{r}\right]\right)=\int_{M\left(r^{0}\right)} \exp \left(\left[\omega_{r^{0}}\right]+\sum_{i=1}^{n}\left(r_{i}-r_{i}^{0}\right) c_{i}\right) .
$$

Then vol $M(r)$ is a polynomial (on each chamber) of degree $n-3$ and

$$
\frac{\partial^{\alpha}}{\partial r^{\alpha}} \operatorname{vol} M(r)_{\left.\right|_{r^{0}}}=\frac{1}{k !} \int_{M\left(r^{0}\right)}\left[\omega_{r^{0}}\right]^{k} c_{1}^{\alpha_{1}} \cdots c_{n}^{\alpha_{n}}
$$

for $\alpha$ multindex, $|\alpha|=\alpha_{1}+\cdots+\alpha_{n}=n-3-k$, with $0 \leq k \leq n-3$.

In particular, if $|\alpha|=n-3$,

$$
\frac{\partial^{\alpha}}{\partial r^{\alpha}} \operatorname{vol} M(r)_{\left.\right|_{r} 0}=\int_{M\left(r^{0}\right)} c_{1}^{\alpha_{1}} \cdots c_{n}^{\alpha_{n}}
$$

If the $c_{i}$ generate the cohomology ring $H^{*}(M(r), \mathbb{C})$, then Guillemin and Sternberg observe that it is possible to read from (5.1) the multiplicative 
relations between the generators, concluding the following explicit description of $H^{*}(M(r), \mathbb{C})$.

Theorem 5.2 ([GS95]). If $c_{1}, \ldots, c_{n}$ generate the cohomology ring $H^{*}(M(r), \mathbb{C})$, then $H^{*}(M(r), \mathbb{C})$ is isomorphic to the abstract ring

$$
\mathbb{C}\left[x_{1}, \ldots, x_{n}\right] / \operatorname{Ann}(\operatorname{vol} M(r)),
$$

where $Q\left(x_{1}, \ldots, x_{n}\right) \in A n n(\operatorname{vol} M(r))$ if and only if $Q\left(\partial / \partial r_{1}, \ldots, \partial / \partial r_{n}\right)$ $\operatorname{vol} M(r)=0$ and the isomorphism is given by $x_{i} \mapsto c_{i}$.

Therefore, it is a central problem to determine when the $c_{i}$ generate the cohomology ring $H^{*}(M(r))$. When $M(r)$ is toric it is well known that this is the case (see, for example, $[\mathbf{F u}]$ ). Still, there are choices of $r$ for which the polygon space $M(r)$ is not toric, as it is the case, for example, for $r=$ $(1,1,1,1,1)$, cf. [HK00] and Remark 2.3.

In general, let $\Delta$ be the set of regular values of $\mu$ in the convex polytope $\Xi$. The connected components $\Delta^{1}, \ldots, \Delta^{\ell}$ of $\Delta$ are themselves convex polytopes. Therefore, by the Duistermaat-Heckman Theorem, the diffeotype of the reduced space $M(r)$ (thus also its cohomology ring) depends only on the chamber $\Delta^{i}$ that contains $r$. If the closure of $\Delta^{i}$ contains a vertex of $\Xi$, then its associated reduced space is a toric manifold and its associated cohomology ring is generated by the $c_{i}$, [GS95].

We prove that this holds for each regular value $r$ by applying the wallcrossing analysis we did in Section 4, showing that crossing a wall has the effect of killing some relations, and so (roughly speaking) some of the generators that were "hidden" appear.

5.2. Wall-crossing and cohomology. By Theorem 4.1, when $r$ crosses the wall $W_{I_{p}}$ the diffeotype of the reduced manifold $M(r)$ changes by replacing a copy of $\mathbb{C P}^{p-2}$ in $M(r)$ by a $\mathbb{C P}^{q-2}$ by means of a blow-up followed by a blow-down.

In this section, we study how the cohomology ring $H^{*}(M(r))$ changes as $r$ crosses a wall; the main tools to prove our result are the Mayer-Vietoris sequence and the Gysin sequence, together with the decomposition theorem as presented in [BBD, CM05].

Let $M$ and $M^{\prime}$ be the moduli spaces of polygons respectively before and after crossing the wall $W_{I_{p}}$. Moreover, denote by $V$ and $V^{\prime}$ the tubular neighborhoods in $M$ and $M^{\prime}$ respectively of the submanifolds as in Theorem 4.1:

$$
\begin{aligned}
V & =N_{\varepsilon} \mathbb{C P}^{p-2}=\text { tubular neighborhood of } \mathbb{C P}^{p-2} \subset M, \\
V^{\prime} & =N_{\varepsilon} \mathbb{C P}^{q-2}=\text { tubular neighborhood of } \mathbb{C P}^{q-2} \subset M^{\prime}, \\
U & =M \backslash \mathbb{C P}^{p-2} \\
U^{\prime} & =M^{\prime} \backslash \mathbb{C P}^{q-2}
\end{aligned}
$$


By the wall-crossing Theorem 4.1, $U=U^{\prime}$ and $U \cap V=U^{\prime} \cap V^{\prime}=: S_{\varepsilon}$. The Mayer-Vietoris sequences for the manifolds $M$ and $M^{\prime}$ are:

$$
\begin{aligned}
& \cdots \rightarrow H^{k-1}\left(S_{\varepsilon}\right) \rightarrow H^{k}(M) \rightarrow H^{k}(U) \oplus H^{k}(V) \rightarrow H^{k}\left(S_{\varepsilon}\right) \rightarrow \cdots \\
& \cdots \rightarrow H^{k-1}\left(S_{\varepsilon}\right) \rightarrow H^{k}\left(M^{\prime}\right) \rightarrow H^{k}\left(U^{\prime}\right) \oplus H^{k}\left(V^{\prime}\right) \rightarrow H^{k}\left(S_{\varepsilon}\right) \rightarrow \cdots
\end{aligned}
$$

Because $H^{k}(U)=H^{k}\left(U^{\prime}\right)$, the change in the cohomology ring structures $H^{*}(M)$ and $H^{*}\left(M^{\prime}\right)$ is enclosed in how $H^{k}\left(V^{\prime}\right)$ and $H^{k}(V)$ map into $H^{k}\left(S_{\varepsilon}\right)$. These maps will be brought to light in the proof of the next proposition.

\section{Proposition 5.3.}

$$
H^{*}\left(S_{\varepsilon}\right)=H^{*}\left(\mathbb{C P}^{\min (p, q)-2}\right) \otimes H^{*}\left(S^{2 \max (p, q)-3}\right)
$$

Proof. By construction, $N_{\varepsilon} \mathbb{C P}^{p-2}$ is the total space of a disk fibration over $\mathbb{C P}^{p-2}$, and $S_{\varepsilon}$ is the total space of the associated fibration in spheres:

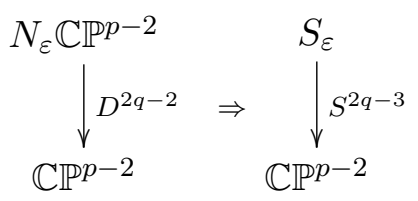

The sphere fibration $\pi: S_{\varepsilon} \stackrel{S^{2 q-3}}{\longrightarrow} \mathbb{C} P^{p-2}$ induces the following Gysin sequence

$$
\rightarrow H^{k}\left(\mathbb{C P}^{p-2}\right) \stackrel{\pi^{*}}{\longrightarrow} H^{k}\left(S_{\varepsilon}\right) \stackrel{\pi_{*}}{\longrightarrow} H^{k-(2 q-3)}\left(\mathbb{C P}^{p-2}\right) \stackrel{\wedge e}{\longrightarrow} H^{k+1}\left(\mathbb{C P}^{p-2}\right) \rightarrow
$$

where $\pi^{*}$ is the map induced in cohomology by the projection map $\pi, \pi_{*}$ is the integration along the fibers and $\wedge e$ is the wedge product with the Euler class.

Recall that

$$
H^{k}\left(\mathbb{C P}^{p-2}\right)= \begin{cases}\mathbb{C} & \text { if } k=0,2, \ldots, 2(p-2), \\ 0 & \text { otherwise. }\end{cases}
$$

Suppose that $q \geq p$. Then the first part of the Gysin map is

$$
\begin{aligned}
& \mathbb{C} \stackrel{\pi^{*}}{\longrightarrow} H^{0}\left(S_{\varepsilon}\right) \stackrel{\pi_{*}}{\longrightarrow} 0 \stackrel{\wedge e}{\longrightarrow} 0 \stackrel{\pi^{*}}{\longrightarrow} H^{1}\left(S_{\varepsilon}\right) \stackrel{\pi_{*}}{\longrightarrow} 0 \stackrel{\wedge e}{\longrightarrow} \mathbb{C} \stackrel{\pi^{*}}{\longrightarrow} \\
& \stackrel{\pi^{*}}{\longrightarrow} H^{2}\left(S_{\varepsilon}\right) \stackrel{\pi_{*}}{\longrightarrow} 0 \stackrel{\wedge e}{\longrightarrow} 0 \stackrel{\pi^{*}}{\longrightarrow} H^{3}\left(S_{\varepsilon}\right) \stackrel{\pi_{*}}{\longrightarrow} 0 \longrightarrow \cdots
\end{aligned}
$$

until cohomology groups of degree $k=2 p-2\left(\right.$ in fact $H^{k-(2 q-3)}\left(\mathbb{C P}^{p-2}\right) \simeq 0$ for all $0 \leq k \leq 2 q-3)$. Therefore,

$$
H^{k}\left(S_{\varepsilon}\right) \simeq H^{k}\left(\mathbb{C P}^{p-2}\right) \quad \forall 0 \leq k \leq 2(p-2) .
$$


At $k=2 q-3$ the Gysin sequence goes as follows:

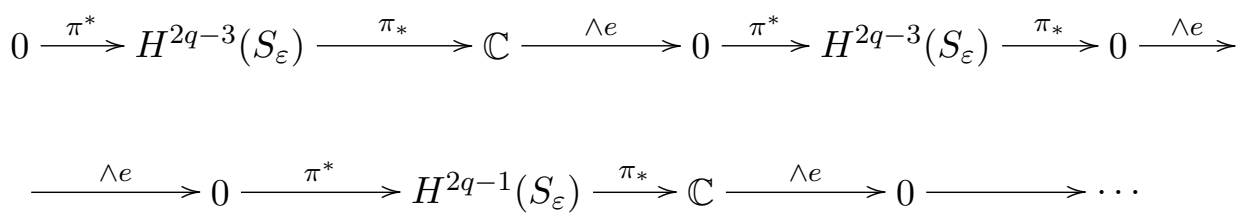

To check this second part the only thing to keep in mind is that $H^{k}\left(\mathbb{C P}^{p-2}\right) \simeq$ 0 for all $k \geq 2 q-3$. In fact $k \geq 2 q-3 \geq 2 p-3>2(p-2)$. Observing that $k-2 q+3=2(p-2) \Longleftrightarrow k=2(p+q)-7$,

$$
\begin{aligned}
& H^{k}\left(S_{\varepsilon}\right) \simeq H^{k-(2 q-3)}\left(\mathbb{C P}^{p-2}\right) \quad \forall 2 q-3 \leq k \leq 2 n-7 . \\
& H^{k}\left(S_{\varepsilon}\right) \simeq 0 \quad \forall k: 2(p-2)<k<2 q-3 \quad \text { or } \quad k \geq 2 n-6 .
\end{aligned}
$$

Summarizing, from (5.2), (5.3) and (5.4), we get

$$
H^{*}\left(S_{\varepsilon}\right)=H^{*}\left(\mathbb{C P}^{p-2}\right) \otimes H^{*}\left(S^{2 q-3}\right) .
$$

It is easy to check that if we assume $p \geq q$ then $p$ and $q$ exchange their roles, and the result follows.

Note that $H^{*}(V)=H^{*}\left(\mathbb{C P}^{p-2}\right)$ because $V$ retracts on $\mathbb{C} P^{p-2}$. Similarly, $H^{*}\left(V^{\prime}\right)=H^{*}\left(\mathbb{C P}^{q-2}\right)$, and we have all the ingredients to write the MayerVietoris sequences for $M$ and $M^{\prime}$ :

$$
\begin{aligned}
H^{0}(M) & \rightarrow H^{0}(U) \oplus \mathbb{C} \rightarrow \mathbb{C} \rightarrow H^{1}(M) \rightarrow H^{1}(U) \oplus 0 \rightarrow 0 \rightarrow \\
& \rightarrow H^{2}(M) \rightarrow H^{2}(U) \oplus \mathbb{C} \rightarrow \mathbb{C} \rightarrow
\end{aligned}
$$

and

$$
\begin{aligned}
H^{0}\left(M^{\prime}\right) & \rightarrow H^{0}\left(U^{\prime}\right) \oplus \mathbb{C} \rightarrow \mathbb{C} \rightarrow H^{1}\left(M^{\prime}\right) \rightarrow H^{1}\left(U^{\prime}\right) \oplus 0 \rightarrow 0 \rightarrow \\
& \rightarrow H^{2}\left(M^{\prime}\right) \rightarrow H^{2}\left(U^{\prime}\right) \oplus \mathbb{C} \rightarrow \mathbb{C} \rightarrow
\end{aligned}
$$

Assume again that $q \geq p$. So, until degree $2(p-2)$, the two sequences above are the same, thus

$$
H^{k}(M)=H^{k}\left(M^{\prime}\right) \quad \forall 0 \leq k \leq 2(p-2) .
$$

At $2(p-2)+1$ the Mayer-Vietoris sequences of the manifolds of $M$ and $M^{\prime}$ are:

$\rightarrow H^{2 p-3}(M) \rightarrow H^{2 p-3}(U) \oplus 0 \rightarrow 0 \rightarrow H^{2 p-2}(M) \rightarrow H^{2 p-2}(U) \oplus 0 \rightarrow 0 \rightarrow$

$\rightarrow H^{2 p-3}\left(M^{\prime}\right) \rightarrow H^{2 p-3}\left(U^{\prime}\right) \oplus 0 \rightarrow 0 \rightarrow H^{2 p-2}\left(M^{\prime}\right) \rightarrow H^{2 p-2}\left(U^{\prime}\right) \oplus \mathbb{C} \rightarrow 0 \rightarrow$

and, until degree $2 q-3$ the two sequences differ by the fact that $H^{k}\left(V^{\prime}\right)=\mathbb{C}$ while $H^{k}(V) \simeq 0$ for $k$ even, $2 p-3 \leq k \leq 2 q-3$. Thus,

$$
\operatorname{dim}\left(H^{k}\left(M^{\prime}\right)\right)=\operatorname{dim}\left(H^{k}(M)\right)+1 \quad \text { if } k \text { even, } 2 p-3 \leq k \leq 2 q-3
$$

and

$$
H^{k}\left(M^{\prime}\right)=H^{k}(M)=0 \quad \text { for } k \text { odd }
$$


At $2 q-3$ the Mayer-Vietoris sequences for $M$ and $M^{\prime}$ are

$$
\begin{aligned}
& \rightarrow H^{2 q-3}(M) \rightarrow H^{2 q-3}(U) \oplus 0 \rightarrow 0 \rightarrow H^{2 q-2}(M) \rightarrow H^{2 q-2}(U) \oplus 0 \rightarrow 0 \rightarrow \\
& \rightarrow H^{2 q-3}\left(M^{\prime}\right) \rightarrow H^{2 q-3}\left(U^{\prime}\right) \oplus 0 \rightarrow 0 \rightarrow H^{2 q-2}\left(M^{\prime}\right) \rightarrow H^{2 q-2}\left(U^{\prime}\right) \oplus 0 \rightarrow 0 \rightarrow
\end{aligned}
$$

and so again (just as for $0 \leq k \leq 2(p-2)$ )

$$
H^{k}\left(M^{\prime}\right) \simeq H^{k}(M) \quad k \geq 2 q-3 .
$$

If $p \geq q$ similar arguments hold, therefore we have proved the following:

\section{Proposition 5.4.}

$$
\begin{array}{ll}
H^{k}\left(M^{\prime}\right)=H^{k}(M)=0, & \text { if } k \text { is odd; } \\
H^{k}\left(M^{\prime}\right)=H^{k}(M), & \left\{\begin{array}{l}
0 \leq k \leq 2(\min (p, q)-2), \\
k \geq 2 \max (p, q)-3 ;
\end{array}\right. \\
\operatorname{dim} H^{k}\left(M^{\prime}\right)=\operatorname{dim} H^{k}(M)+1 \quad k \text { even, } 2 p-2 \leq k \leq 2 q-4(\text { case } q \geq p) ; \\
\operatorname{dim} H^{k}(M)=\operatorname{dim} H^{k}\left(M^{\prime}\right)+1 \quad k \text { even, } 2 q-2 \leq k \leq 2 p-4(\text { case } p \geq q) ;
\end{array}
$$

This calculation, done using the Mayer-Vietoris sequences of the manifolds $M$ and $M^{\prime}$, tells us in which degree the cohomology groups of the symplectic quotient $M(r)$ change as $r$ crosses a wall $W_{I_{p}}$.

Even though it is natural- by the construction- to expect that the new born cohomological classes are polynomial in the class of the blown up manifold $\mathbb{C P}^{q-2}$ or $\mathbb{C P}^{p-2}$, this calculation does not give us such precise informations. We use the decomposition Theorem due to BeilinsonBernstein-Deligne $[\mathbf{B B D}]$ to identify precisely the new born classes that increase the dimension of the cohomology groups of "middle" degrees. To this aim, some notation needs to be introduced.

Let $f: X \rightarrow Y$ be a map of algebraic manifolds (i.e., manifolds which are the set of common zeros of a finite number of polynomials). For each $0<k<\frac{\operatorname{dim} X}{2}$, define $Y_{k}:=\left\{y \in Y: \operatorname{dim}\left(f^{-1}(y)\right) \geq k\right\}$. The map $f$ is small if and only if

$$
\operatorname{dim} Y_{k}+2 k<\operatorname{dim} X \quad \forall 0<k<\frac{\operatorname{dim} X}{2}
$$

and semi-small if and only if

$$
\operatorname{dim} Y_{k}+2 k \leq \operatorname{dim} X \quad \forall 0<k<\frac{\operatorname{dim} X}{2} .
$$

Proposition 5.5. At least one of the blow-down maps $\beta_{+}$and $\beta_{-}$as in Theorem 4.1 is small.

Proof. Denote by $Y_{k}^{ \pm}:=\left\{y \in M\left(r^{c}\right): \operatorname{dim}\left(\beta_{ \pm}^{-1}(y)\right) \geq k\right\}$. If $q>p$, then $\beta_{+}$ is small. In fact

$$
Y_{k}^{+}= \begin{cases}\left\{\left[P^{c}\right]\right\} & \text { for } 1 \leq k \leq 2(p-2) \\ \emptyset & \text { otherwise }\end{cases}
$$


Thus, inequality (5.5) for $k=2(p-2)$ is verified:

$$
4(p-2)<2(n-3) \Longleftrightarrow 4 p-8<2 p+2 q-6 \Longleftrightarrow p-1<q,
$$

and similarly inequality (5.5) is verified for smaller $k$ 's. Under the assumption $q>p$ the map $\beta_{-}$is not semi-small (thus even not small). In fact $Y_{k}^{-}=\left\{\left[P^{c}\right]\right\}$ and inequality (5.6) for $k=q-2$ does not hold since

$$
4(q-2) \leq 2(n-3) \Longleftrightarrow q-1 \leq p .
$$

If $p>q$, then $\beta_{-}$is small and $\beta_{+}$is not semi-small. Note that if $p=q$ then both $\beta_{-}$and $\beta_{+}$are small.

Assume that $\beta_{+}: M \rightarrow M\left(r^{c}\right)$ is small. Then $H^{*}(M)=I H^{*}\left(M\left(r^{c}\right)\right)$, where $I H^{*}\left(M\left(r^{c}\right)\right)$ is the intersection cohomology of the singular manifold $M\left(r^{c}\right)$ (see the survey paper by de Cataldo and Migliorini $[\mathbf{C M}]$ ).

We state the decomposition Theorem just for the special situation of $\beta_{+}$ and $\beta_{-}$resolutions of the singularity corresponding to the lined polygon in $M\left(r^{c}\right)$. For the statement in full generality, proofs and more details we refer to the original paper $[\mathbf{B B D}]$, and to $[\mathbf{C M 0 5}]$ by de Cataldo-Migliorini, where an alternative proof is given.

In our setting, the decomposition Theorem says that $H^{*}\left(M^{\prime}\right)$ is isomorphic to the intersection cohomology $I H^{*}\left(M\left(r^{c}\right)\right)$ of $M\left(r^{c}\right)$ plus polynomials in the cohomological classes of submanifolds $\mathcal{C}_{i}$ of $M$. In the moduli space situation, these submanifolds are just the preimages of the points $y_{i} \in Y_{k}^{+}$.

If we assume that $q \geq p$ (which is equivalent to assuming $\beta_{+}$small), then $C:=\left(\beta_{-}\right)^{-1}\left(\left[P^{c}\right]\right)$ is the resolution in $M^{\prime}$ of the singularity $\left[P^{c}\right]$. By Theorem 4.1,

$$
\mathcal{C}=M_{I_{p}}(r) \simeq \mathbb{C P}^{q-2} .
$$

Applying the decomposition Theorem we get:

Theorem 5.6. Let $\beta_{+}: M \rightarrow M\left(r^{c}\right)$ be a small resolution of the singularity in $M\left(r^{c}\right)$ and let $M^{\prime}$ be the polygon space birational to $M$ via the single wallcrossing described above. Then

$$
H^{*}\left(M^{\prime}\right)=H^{*}(M) \oplus \bigoplus_{\alpha=0}^{q-p} \mathbb{C}\left(P D\left(\left[M_{I_{p}}(r)\right]\right) \smile c_{1}^{\alpha}\left(\mathcal{N}^{\prime}\right)\right)
$$

where $P D\left(\left[M_{I_{p}}(r)\right]\right) \in H^{2 p-2}\left(M^{\prime}\right)$ is the Poincaré dual of $M_{I_{p}}(r) \subset M^{\prime}$, and $c_{1}\left(\mathcal{N}^{\prime}\right)$ is the first Chern class of the normal bundle $\mathcal{N}^{\prime}$ to $M_{I_{p}}(r) \subseteq M^{\prime}$.

At the light of this result, to prove that $H^{*}(M(r))$ is generated by the Chern classes $c_{i}$ we need to express the classes $P D\left(\left[M_{I_{p}}(r)\right]\right)$ and the cup products $P D\left(\left[M_{I_{p}}(r)\right]\right) \smile c_{1}^{\alpha}\left(\mathcal{N}^{\prime}\right)$ as combinations of the $c_{i}$. 
By Poincaré duality, $P D\left(\left[M_{I_{p}}(r)\right]\right) \in H^{2 p-2}\left(M^{\prime}\right)$ and thus we want to show that, for some constants $A_{\alpha}$,

$$
P D\left(\left[M_{I_{p}}(r)\right]\right)=\sum_{\sum \alpha_{i}=p-1} A_{\alpha} c_{1}^{\alpha_{1}} \cdots c_{n}^{\alpha_{n}} .
$$

To this aim, we will explicitly describe the classes $c_{i}$ by means of the two description of the polygon space $M(r)$ that one gets from the $U(1)^{n} \times U(2)$ action on $\mathbb{C}^{n \times 2}$ by performing reduction in stages as summarized in diagram 2.3 .

Let $r$ be a regular value in $\Delta^{1}$ such that the reduced manifold $M(r) \simeq M^{\prime}$. Since the fibration $\mu^{-1}(r) \rightarrow M(r)$ as in (2.3) is trivial, the classes $c_{i}$ of the $n$ complex line bundles associated to it are actually the classes $c_{i}$ relative to the $n$ Hopf fibrations $S_{\sqrt{2 r_{i}}}^{3} \rightarrow S_{r_{i}}^{2}$. These are well known to be the Chern classes of the tautological line bundle $\mathcal{O}(-1)$ over $\mathbb{C P}^{1}$ (under the identification $\mathbb{C P}^{1} \simeq S^{2}$. More precisely,

$$
s^{*} c_{i}=c_{1}\left(p_{i}^{*}(\mathcal{O}(-1))\right)=-\left[p_{i}^{*} \omega_{\mathrm{FS}}\right],
$$

where $s$ is the fibration $s: \mu_{S O(3)}^{-1}(0) \rightarrow M(r), p_{i}$ is the projection $p_{i}$ : $\prod_{j} S_{r_{j}}^{2} \rightarrow S_{r_{i}}^{2}$ and $\omega_{\mathrm{FS}}$ is the Fubini-Study symplectic form.

Proposition 5.7. The Poincaré dual of $M_{I_{p}}(r)$ is the $(2 p-2)$-class

$$
P D\left(\left[M_{I_{p}}(r)\right]\right)=(-1)^{p-1} \prod_{j=2, \ldots, p}\left(c_{i_{j}}+c_{i_{1}}\right) \in H^{2 p-2}\left(M^{\prime}\right)
$$

where $I_{p}=\left\{i_{j} \mid j=1, \ldots, p\right\}$. Moreover first Chern class $c_{1}\left(\mathcal{N}^{\prime}\right)$ of the normal bundle to $M_{I_{p}}(r)$ is

$$
c_{1}\left(\mathcal{N}^{\prime}\right)=-2\left(c_{i_{2}}+\cdots+c_{i_{p}}\right) .
$$

Proof. The polygon space $M(r)$ can also be described as the GIT quotient of $\prod_{n} \mathbb{C P}^{1}$ by the diagonal action of $\operatorname{PSL}(2, \mathbb{C})$. Let $H \rightarrow \mathbb{C P}^{1}$ be the hyperplane bundle. Then the line bundle $p_{i}^{*} H \otimes p_{j}^{*} H$ over $\prod_{n} \mathbb{C P}^{1}$ induces a line bundle $L_{i j}$ on the quotient $M(r)$, cf $[\mathbf{K o}]$. For all $i=1, \ldots, n$, let $z_{i}$ be the first Chern class

$$
z_{i}:=c_{1}\left(L_{i i}\right) \in H^{2}(M(r), \mathbb{Z}) .
$$

Clearly $s^{*} z_{i}=c_{1}\left(p_{i}^{*} \mathcal{O}(2)\right)=2\left[p_{i}^{*} \omega_{\mathrm{FS}}\right]$. It follows that

$$
z_{i}=-2 c_{i} .
$$

In the equilateral case, Kamiyama and Tezuka $[\mathbf{K T}]$ prove that the Poincaré dual of $\frac{z_{i}+z_{j}}{2}$ is the submanifold of $M(1, \ldots, 1)$ consisting of those polygons $\vec{e}$ such that $e_{i}=e_{j}$. This easily generalizes to the non-equilateral case 
(cf. $[\mathbf{K o}])$, and the Poincaré dual of $\frac{z_{i}+z_{j}}{2}=-\left(c_{i}+c_{j}\right)$ is the submanifold $M_{\{i, j\}}(r)$. Since

$$
M_{I_{p}}(r)=\bigcap_{i_{j} \in I_{p} \backslash\left\{i_{1}\right\}} M_{\left\{i_{1}, i_{j}\right\}}(r)
$$

the result follows.

Analogously one can prove that the first Chern class $c_{1}\left(\mathcal{N}^{\prime}\right)$ of the normal bundle to $M_{I_{p}}(r)$ is a linear combination of the Chern classes $c_{i}, i=1, \ldots, n$. In fact, the tangent bundle to $M(r)$ is the direct sum $p_{1}^{*} \mathcal{O}(2) \oplus \cdots \oplus p_{n}^{*} \mathcal{O}(2)$ of the pullbacks of the tangent bundle to each sphere.

The submanifold $M_{I_{p}}(r)$ is the moduli space of polygons obtained as the quotient by the $S O(3)$-action on the product of spheres of radii $\left(\sum_{i \in I_{p}} r_{i}, r_{i_{p+1}}, \ldots, r_{i_{n}}\right)$, with $I_{p}=\left\{i_{1}, \ldots, i_{p}\right\}$. Since the inclusion $S_{\sum_{i \in I_{p}} r_{i}}^{2} \hookrightarrow S_{r_{i_{1}}}^{2} \times \cdots \times S_{r_{i_{p}}}^{2}$ is the diagonal one, then the tangent bundle to $M_{I_{p}}(r)$ is

$$
\left(p_{i_{1}}^{*} \mathcal{O}(2) \otimes \cdots \otimes p_{i_{p}}^{*} \mathcal{O}(2)\right) \oplus p_{i_{p+1}}^{*} \mathcal{O}(2) \oplus \cdots \oplus p_{i_{n}}^{*} \mathcal{O}(2)
$$

and therefore the first Chern class of the quotient $T M(r) / T M_{I_{p}}(r)$ is the sum of $(p-1)$ among the first Chern classes $c_{i}$ for $i \in I_{p}$, i.e.,

$$
c_{1}\left(\mathcal{N}^{\prime}\right)=-2\left(c_{i_{2}}+\cdots+c_{i_{p}}\right) .
$$

Note that similar arguments hold if $p>q$, i.e., if $\beta_{+}$is not small while $\beta_{-}$ is. In this case, the decomposition Theorem implies that the cohomology of $M$ is described as follows

$$
H^{*}(M)=H^{*}\left(M^{\prime}\right) \oplus \bigoplus_{\alpha=0}^{p-q} \mathbb{C}\left(P D\left(\left[M_{I_{q}}(r)\right]\right) \smile c_{1}^{\alpha}(\mathcal{N})\right),
$$

where $P D\left(\left[M_{I_{q}}(r)\right]\right) \in H^{2 q-2}(M)$ is the class of $M_{I_{q}}(r) \subset M$, and $c_{1}(\mathcal{N})$ is the first Chern class of the normal bundle $\mathcal{N}$ to $M_{I_{q}}(r) \subseteq M$. Moreover $P D\left(\left[M_{I_{q}}(r)\right]\right) \in H^{2 q-2}(M)$ and $c_{1}(\mathcal{N})$ are clearly combinations of the $c_{i}$ 's, since Proposition 5.7 follows from diagram (2.3) (which holds for any smooth polygon space, and in particular for any $r$ such that $M(r) \simeq M)$. Thus, by Theorem 5.2, the following holds:

Theorem 5.8. For $r$ generic, the cohomology ring $H^{*}(M(r), \mathbb{C})$ of the moduli space of polygons $M(r)$ is generated by the first Chern classes $c_{1}, \ldots, c_{n}$ of the $n$ complex line bundles associated to the fibration $\mu^{-1}\left(r_{1}, \ldots, r_{n}\right) \rightarrow$ $M(r)$. So

$$
H^{*}(M(r), \mathbb{C}) \simeq \mathbb{C}\left[x_{1}, \ldots, x_{n}\right] / \operatorname{Ann}(\operatorname{vol} M(r))
$$


where a polynomial $Q\left(x_{1}, \ldots, x_{n}\right) \in A n n(\operatorname{vol} M(r))$ if and only if

$$
Q\left(\frac{\partial}{\partial r_{1}}, \ldots, \frac{\partial}{\partial r_{n}}\right) \operatorname{vol} M(r)=0
$$

and, as in Theorem 5.2, the isomorphism is given by $x_{i} \mapsto c_{i}$.

Note that the formula (5.1) determines not only the cohomology ring of the polygon space $M(r)$ but also its intersection numbers. Explicit formulas for these have been obtained by Agapito and Godinho $[\mathbf{A G}]$ via a recursion relation in $n$, by Takakura [Ta01] using "quantization commutes with reduction" and by Konno [Ko] using algebro-geometric methods. For example, consider the lengths vector $r=(4,3,4,3,4)$ as in [AG, Example 7.1] (or equivalently its projection onto $\Xi$ ). By Theorem 3.4 one calculates that the volume of $M(r)$ is

$$
\operatorname{vol} M(r)=-\pi^{2}\left(6 \sum_{i=1}^{n} r_{i}^{2}-2 \sum_{i \neq j} r_{i} r_{j}\right) .
$$

By the formulas (5.1) one recovers (up to rescaling by $2 \pi^{2}$ ) the results in $[\mathbf{A G}]$, precisely

$$
\begin{gathered}
\int_{M(r)} c_{i}^{2}=-6 \pi^{2} \quad \forall i=1, \ldots, 5 ; \\
\int_{M(r)} c_{i} c_{j}=2 \pi^{2} \quad \forall i \neq j .
\end{gathered}
$$

Remark 5.9. In [HK98] Hausmann and Knutson compute the cohomology ring $H^{*}(M(r), \mathbb{Z})$ in terms of generators they call $R$ and $V_{i}$. Denote by $\mathcal{L}$ the collection of $r$-long sets and define the collection of indeces $\mathcal{L}_{n}$ and $\mathcal{S}_{n}$ as follows:

$$
\begin{aligned}
& \mathcal{L}_{n}:=\{J \subset\{1, \ldots, n-1\} \mid J \cup\{n\} \text { is long }\} \\
& \mathcal{S}_{n}:=\{J \subset\{1, \ldots, n-1\} \mid J \cup\{n\} \text { is short }\} .
\end{aligned}
$$

Theorem 5.10 ((Hausmann-Knutson)). For $r$ generic, the cohomology ring $H^{*}(M(r), \mathbb{Z})$ is

$$
\mathbb{Z}\left[R, V_{1}, \ldots, V_{n-1}\right] / I_{\mathrm{Pol}}
$$

where $R$ and $V_{i}$ are of degree 2 and $I_{\mathrm{Pol}}$ is generated by the following three families:

- $V_{i}^{2}+R_{i} V_{i}$ for all $i=1, \ldots n-1$;

- $\prod_{i \in J} V_{i}$ for all $J \in \mathcal{L}_{n}$;

- $\sum_{S \subset L, S \in S_{n}}\left(\prod_{i \in S} V_{i}\right) R^{|L \backslash S|-1}$ for all $L \subset\{1, \ldots, n-1\}$ long. 
They also relate the generators $R$ and $V_{i}$ to the first Chern classes $\tilde{c}_{i}:=$ $c_{1}\left(A_{i}\right)$ of circle bundles $A_{i} \rightarrow M(r)$ where

$$
A_{i}:=\left\{\left(e_{1}, \ldots, e_{n}\right) \in \prod_{i=1}^{n} S_{r_{i}}^{2} \mid \sum_{i=1}^{n} e_{i}=0 \text { and } e_{i}=\left(0,0, r_{i}\right)\right\} .
$$

Precisely,

$$
\tilde{c}_{i}= \begin{cases}R+2 V_{i} & \text { if } i=1, \ldots, n-1 \\ -R & \text { if } i=n .\end{cases}
$$

Let $\omega$ be the reduced symplectic form on the polygon space $M(r)$. Then

$$
\tilde{c}_{i}=\frac{\partial}{\partial r_{i}}[\omega]
$$

Hence, by (5.7), the Chern classes $c_{i}$ in Theorem 5.8 are opposite to the classes $\tilde{c}_{i}$, i.e., $c_{i}=-\tilde{c}_{i}$. In particular, this implies that the classes $c_{1}, \ldots, c_{n}$ also generate the cohomology of the polygon space $M(r)$ with coefficients in $\mathbb{Z}\left[\frac{1}{2}\right]$, cf. [HK98, Corollary 7.4, Proposition 7.6]. Hausmann and Knutson determine the following relations on the generators $c_{i}$ :

(1) $c_{i}^{2}=c_{n}^{2}$ for all $i=1, \ldots, n$;

(2) $\prod_{i \in L}\left(c_{i}+c_{n}\right)$ for all $L \in \mathcal{L}_{n}$;

(3) $c_{n}^{-1}\left(\prod_{i \in L}\left(c_{i}-c_{n}\right)-\prod_{i \in L}\left(c_{i}+c_{n}\right)\right)$ for all $L \subseteq\{1, \ldots, n-1\}, L$ long.

The relations (1) may also be easily obtained from Theorems 5.8 and 3.4 since

where

$$
\frac{\partial}{\partial r_{i}} \varepsilon_{I}(r)^{n-3}=\lambda_{I}^{i}(n-3) \varepsilon_{I}(r)^{n-2}
$$

5.2.1. Some examples. Let $\Delta^{0}$ and $\Delta^{1}$ be the chambers as in Example 3.3. If $r \in \Delta^{0}$, then $M(r) \simeq \mathbb{C P}^{2}$ (see Proposition 4.2) and its symplectic volume is

$$
\operatorname{vol} M(r)=2 \pi^{2}\left(r_{1}+r_{2}-r_{3}+r_{4}+r_{5}\right)^{2} .
$$

Since

it follows that

$$
\frac{\partial}{\partial r_{3}} \operatorname{vol} M(r)=-\frac{\partial}{\partial r_{i}} \operatorname{vol} M(r) \quad \forall i=1,2,4,5
$$

$$
c_{1}=c_{2}=c_{4}=c_{5}=-c_{3} .
$$

By Theorem 5.8, the relation on the generator $c_{3}$ is given by $\frac{\partial^{2}}{\partial r_{3}^{2}} \operatorname{vol} M(r)=$ $4 \pi^{2}$, hence

$$
H^{*}(M(r))=\frac{\mathbb{C}\left[c_{3}\right]}{\left(c_{3}^{3}\right)}
$$


Now consider $r \in \Delta^{1}$, the polygon space $M(r)$ has symplectic volume

$$
\operatorname{vol} M(r)=4 \pi^{2} r_{1}\left(r_{2}-r_{3}+r_{4}+r_{5}\right) .
$$

It follows that

$$
\frac{\partial}{\partial r_{3}} \operatorname{vol} M(r)=-\frac{\partial}{\partial r_{i}} \operatorname{vol} M(r) \quad \forall i=2,4,5
$$

and hence

$$
c_{2}=c_{4}=c_{5}=-c_{3} .
$$

The relations on the Chern classes $c_{1}$ and $c_{3}$ are given by

$$
\frac{\partial^{2}}{\partial r_{1}^{2}} \operatorname{vol} M(r)=0 \quad \text { and } \quad \frac{\partial^{2}}{\partial r_{3}^{2}} \operatorname{vol} M(r)=0
$$

Hence, the cohomology ring of $M(r)$ is

$$
H^{*}(M(r))=\frac{\mathbb{C}\left[c_{1}, c_{3}\right]}{\left(c_{1}^{2}, c_{3}^{2}\right)} .
$$

By the wall-crossing study, cf. Section $4.1, M(r)$ is diffeomorphic to $\mathbb{C P}^{2}$ blown up at a point with exceptional divisor $M_{\{1,3\}}$. By Proposition 5.7

$$
P D\left(\left[M_{\{1,3\}}(r)\right]\right)=-\left(c_{1}+c_{3}\right) .
$$

With respect to the basis $\left\{-c_{1}+c_{3},-\left(c_{1}+c_{3}\right)\right\}$ the polygon space $M(r)$ has intersection form $\left(\begin{array}{cc}-1 & 0 \\ 0 & 1\end{array}\right)$.

Consider now the lengths vector $r=\frac{1}{11}(3,1,3,1,3)$. Hausmann and Knutson [HK97] have shown that $M(r)$ is isomorphic to $S^{2} \times S^{2}$. It is again a plain computation to obtain the volume of $M(r)$ :

$$
\operatorname{vol} M(r)=8 \pi^{2} r_{2} r_{4}
$$

From this we obtain the relations

$$
c_{1}=c_{3}=c_{5}=0
$$

and by Theorem 5.8 the cohomology of $M(r)$ is

$$
H^{*}(M(r))=\frac{\mathbb{C}\left[c_{2}, c_{4}\right]}{\left(c_{2}^{2}, c_{4}^{2}\right)} .
$$

Moreover, with respect to the basis $\left\{c_{2}, c_{4}\right\}$ the polygon space $M(r)$ has intersection form $\left(\begin{array}{ll}1 & 0 \\ 0 & 1\end{array}\right)$. This also illustrates that $H^{*}\left(\mathbb{C P}^{2} \sharp \overline{\mathbb{C P}^{2}}, \mathbb{C}\right) \approx$ $H^{*}\left(S^{2} \times S^{2}, \mathbb{C}\right)$ as indeed the intersection forms $\left(\begin{array}{cc}-1 & 0 \\ 0 & 1\end{array}\right)$ and $\left(\begin{array}{ll}1 & 0 \\ 0 & 1\end{array}\right)$ are equivalent over $\mathbb{C}$ (in fact, they are equivalent over $\mathbb{Z}\left[\frac{1}{2}\right]$ ). 
The latter example is a particular case of lengths vectors of type

$$
r=\frac{1}{p}\left(r_{1}, \ldots, r_{n-3}, 1,1,1\right)
$$

with $\sum_{i=1}^{n-3} r_{i}<1$ and $p=3+\sum_{i=1}^{n-3} r_{i}$. In this case, the long sets $I$ are all and just the sets that contain at least two elements of $\{n-2, n-1, n\}$. The volume of the associated polygon space $M(r)$ is

$$
\operatorname{vol} M(r)=\frac{(2 \pi)^{n-3}}{(n-3) !} 2^{n-2} r_{1} \cdots r_{n-3} .
$$

Thus, $c_{n-2}=c_{n-1}=c_{n}=0$ and

$$
H^{*}(M(r))=\frac{\mathbb{C}\left[c_{1}, \ldots, c_{n-3}\right]}{\left(c_{1}^{2}, \ldots, c_{n-3}^{2}\right)} .
$$

To obtain (5.10) from Theorem 3.4 one can first observe that the volume of $M(r)$ can be rewritten as follows:

$$
\begin{aligned}
\operatorname{vol} M(r) & =C \sum_{I \text { long }}(-1)^{n-|I|} \sum_{\left(k_{1}, \ldots, k_{n}\right) \in K}\left(\begin{array}{c}
n-3 \\
k_{1}, \ldots, k_{n}
\end{array}\right)\left(\lambda_{I}^{1} r_{1}\right)^{k_{1}} \cdots\left(\lambda_{I}^{n} r_{n}\right)^{k_{n}} \\
& =C \sum_{\left(k_{1}, \ldots, k_{n}\right) \in K}\left(\begin{array}{c}
n-3 \\
k_{1}, \ldots, k_{n}
\end{array}\right) r_{1}^{k_{1}} \cdots r_{n}^{k_{n}} \sum_{I \text { long }}(-1)^{n-|I|}\left(\lambda_{I}^{1}\right)^{k_{1}} \cdots\left(\lambda_{I}^{n}\right)^{k_{n}}
\end{aligned}
$$

where $C=-\frac{(2 \pi)^{n-3}}{2(n-3) !}, K=\left\{\left(k_{1}, \ldots, k_{n}\right) \in \mathbb{Z}_{+}^{n} \mid \sum_{i=1}^{n} k_{i}=n-3\right\}$ and $\lambda_{I}^{i}$ is as in (5.9). Let us concentrate on the second sum.

For $\left(k_{1}, \ldots, k_{n}\right)=(1, \ldots, 1,0,0,0)$ one obtains

$$
\sum_{I \text { long }}(-1)^{n-|I|} \lambda_{I}^{1} \cdots \lambda_{I}^{n}
$$

$$
\begin{aligned}
& =3 \sum_{j=0}^{n-3}\left(\begin{array}{c}
n-3 \\
j
\end{array}\right)(-1)^{n-2-j}(-1)^{n-3+j}+\sum_{j=0}^{n-3}\left(\begin{array}{c}
n-3 \\
j
\end{array}\right)(-1)^{n-3-j}(-1)^{n-3+j} \\
& =2 \sum_{j=0}^{n-3}\left(\begin{array}{c}
n-3 \\
j
\end{array}\right)=-2^{n-3}
\end{aligned}
$$

where the first sum in (5.11) is relative to long sets $I$ such that $\mid I \cap\{n-2, n-$ $1, n\} \mid=2$ and the second sum to long sets $I$ such that $\{n-2, n-1, n\} \subseteq I$. By similar arguments one can prove that for any other choice of $\left(k_{1}, \ldots, k_{n}\right) \in K$ one obtains

$$
\sum_{I \text { long }}(-1)^{n-|I|}\left(\lambda_{I}^{1}\right)^{k_{1}} \cdots\left(\lambda_{I}^{n}\right)^{k_{n}}=0,
$$

hence proving (5.10). 


\section{References}

[AG] J. Agapito and L. Godinho, Intersection numbers of polygon spaces, Trans. Amer. Math. Soc. 361(9) (2009), 4969-4997.

[AB] M.F. Atiyah and R. Bott The moment map and equivariant cohomology, Topology 23(1) (1984), 1-28.

[Au] M. Audin The topology of torus action on symplectic manifold, Birkhäuser, Basel, 1991.

[BBD] A.A. Beilinson, J. Bernstein and P. Deligne, Faisceaux pervers, Astérisque, 100 (1982).

[B] M. Brion, Cohomologie équivariante des points semi-stables, J. Reine Angew. Math. 421 (1991), 125-140.

[BP] M. Brion and C. Procesi, Action d'un tore dans une variété projective, Operator algebras, unitary representations, enveloping algebras, and invariant theory (Paris, 1989), 509-539, Progr. Math., 92, Birkhäuser Boston, Boston, MA, 1990.

[CM05] M.A.A. de Cataldo and L. Migliorini, The Hodge theory of algebraic maps, Ann. Sci. École Norm. Sup. (4) 38(5) (2005), 693-750.

[CM] M.A.A. de Cataldo and L. Migliorini, Intersection forms, topology of maps and motivic decomposition for resolutions of threefolds, Algebraic cycles and motives, Vol. 1, 102-137, London Math. Soc. Lecture Note Ser., 343, Cambridge University Press, Cambridge, 2007.

[Du] J.J. Duistermaat, Equivariant cohomology and stationary phase, Symplectic geometry and quantization (Sanda and Yokohama, 1993), 45-62, Contemp. Mathematics, 179, Amer. Math. Soc., Providence, RI, 1994.

$[\mathrm{DH}]$ J.J. Duistermaat and G.J. Heckman, On the variation of the cohomology of the symplectic form of the reduced phase space, Invent. Math. 69(2) (1982), 259-268

[FHS] M. Farber, J.C. Hausmann and D. Schütz, On the conjecture of Kevin Walker, J. Topol. Anal. 1(1) (2009), 65-86.

[Fu] W. Fulton, Introduction to toric varieties, in 'Annals of Mathematics Studies', 131; Princeton University Press, Princeton, NJ, 1993.

[GM] I.M. Gelfand and R.D. MacPherson, Geometry in Grassmannians and a generalization of the dilogarithm, Adv. Math. 44(3) (1982), 279-312.

[Go] R.F. Goldin, The cohomology ring of weight varieties and polygon spaces, Adv. Math. 160(2) (2001), 175-204.

[Gu] V. Guillemin, Moment maps and combinatorial invariants of Hamiltonian $T^{n}$ spaces, Progress in Mathematics, 122, Birkhäuser Boston, Inc., Boston, MA, 1994, viii+150 pp.

[GK] V. Guillemin and J. Kalkman, The Jeffrey-Kirwan localization theorem and residue operations in equivariant cohomology, J. Reine Angew. Math. 470 (1996), 123-142.

[GS89] V. Guillemin and S. Sternberg, Birational equivalence in the symplectic category, Invent. Math. 97(3) (1989), 485-522.

[GS95] V. Guillemin and S. Sternberg, The coefficients of the Duistermaat-Heckman polynomial and the cohomology ring of reduced spaces, Geometry, topology and physics, 202-213, Conf. Proc. Lecture Notes Geom. Topology, IV, International Press, Cambridge, MA, 1995. 
[HK00] J.C. Hausmann and A. Knutson, A limit of toric symplectic forms that has no periodic Hamiltonians, Geom. Funct. Anal. 10(3) (2000), 556-562.

[HK98] J.C. Hausmann and A. Knutson, The cohomology ring of polygon spaces, Ann. Inst. Fourier (Grenoble) 48(1) (1998), 281-321.

[HK97] J.C. Hausmann and A. Knutson, Polygon spaces and Grassmannians, Enseign. Math. (2) 43(1-2) (1997), 173-198.

[KT] Y. Kamiyama and M. Tezuka, Symplectic volume of the moduli space of spatial polygons, J. Math. Kyoto University 39(3) (1999), 557-575.

[KM] M. Kapovich and J.J. Millson, The symplectic geometry of polygons in Euclidean space, J. Differ. Geom. 44(3) (1996), 479-513.

[Kh] V.T. Khoi, On the symplectic volume of the moduli space of spherical and Euclidean polygons, Kodai Math. J. 28(1) (2005), 199-208.

[Ki92] F. Kirwan, The cohomology rings of moduli spaces of bundles over Riemann surfaces, J. Amer. Math. Soc. 5(4) (1992), 853-906.

[Ki] F.C. Kirwan, Cohomology of quotients in symplectic and algebraic geometry, Mathematical Notes, 31. Princeton University Press, Princeton, NJ, 1984.

[Kl] A. Klyachko, Spatial polygons and stable configurations of points in the projective line, Algebraic geometry and its applications (Yaroslavl', 1992), 67-84, Aspects Math., E25, Vieweg, Braunschweig, 1994.

[Ko] H. Konno, The intersection pairings on the configuration spaces of points in the projective line, J. Math. Kyoto University 41(2) (2001), 277-284.

[Ma] S.K. Martin, Transversality theory, cobordisms, and invariants of symplectic quotients, from $\mathrm{PhD}$ thesis, arXiv:math/0001001.

[Ma2] S.K. Martin, Symplectic quotients by a nonabelian group and by its maximal torus, from PhD thesis, arXiv:math/0001002.

[M] A. Mandini, The cobordism class of the moduli space of polygons in $\mathbb{R}^{3}$, J. Symplectic Geom. 7(1) (2009), 1-27.

[Ta01] T. Takakura, Intersection theory on symplectic quotients of products of spheres, Int. J. Math. 12(1) (2001), 97-111.

[Ta02] T. Takakura, A note on the symplectic volume of the moduli space of spatial polygons, Minimal surfaces, geometric analysis and symplectic geometry (Baltimore, MD, 1999), 255-259, Adv. Stud. Pure Math., 34, Math. Soc. Japan, Tokyo, 2002.

Center for Mathematical Analysis

Geometry and Dynamical Systems

Departamento de Matematica

Instituto Superior TECNico

1049-001 LisBOA

Portugal

E-mail address: amandini@math.ist.utl.pt

Mathematics Department

UNIVERSITY OF PAVIA

VIA FERRATA 1

27100 PaVia (ItAly)

E-mail address: alessia.mandini@gmail.com 
Received 09/15/2010, accepted 03/27/2013

I am grateful to Luca Migliorini for the insightful discussions and support. I also wish to thank ICTP in Trieste and CRM "Ennio de Giorgi" in Pisa for providing excellent working conditions that made these discussions possible. I also wish to thank Leonor Godinho for helpful suggestions, and the University of Utrecht for its hospitality. Finally I would like to thank the referees for several improving comments.

Partially supported by the Fundação para a Ciência e a Tecnologia (FCT /Portugal), grant SFRH / BPD / 44041 / 2008 and by Centro de Análise Matemática, Geometria e Sistemas Dinâmicos, Departamento de Matemática, IST, Lisbon (Portugal).

The research leading to these results has received funding from the European Research Council under the European Union's Seventh Framework Programme (FP7/2007-2013) / ERC Grant agreement no. 307119. 
